\title{
Metal-containing nanomaterials as lubricant additives: State-of-the-art and future development
}

\author{
Igor E. UFLYAND ${ }^{1, *}$, Vladimir A. ZHINZHILO ${ }^{1}$, Victoria E. BURLAKOVA ${ }^{2}$ \\ ${ }^{1}$ Department of Chemistry, Southern Federal University, Rostov-on-Don 344006, Russian Federation \\ ${ }^{2}$ Department of Chemistry, Don State Technical University, Rostov-on-Don 344010, Russian Federation \\ Received: 18 August 2018 / Revised: 27 October 2018 / Accepted: 28 November 2018 \\ (C) The author(s) 2019.
}

\begin{abstract}
This review focuses on the effect of metal-containing nanomaterials on tribological performance in oil lubrication. The basic data on nanolubricants based on nanoparticles of metals, metal oxides, metal sulfides, nanocomposities, and rare-earth compounds are generalized. The influence of nanoparticle size, morphology, surface functionalization, and concentration on friction and wear is analyzed. The lubrication mechanisms of nanolubricants are discussed. The problems and prospects for the development of metal-containing nanomaterials as lubricant additives are considered. The bibliography includes articles published during the last five years.
\end{abstract}

Keywords: coefficient of friction; metal-containing nanomaterials; nanolubricants; nanoparticles; wear

\section{Introduction}

Recently, the use of nanomaterials as lubricant additives (also known as nanolubricants) has become an important research area [1-18]. The nanolubricant approach is used to overcome the drawbacks of conventional anti-wear and anti-friction additives associated with the need for chemical reactions with substrates, and hence the induction period for obtaining a tribo-film on the friction surface. The main advantages of nanoparticles (NPs) are their size in the nanometer range, which is well adapted for the ideal filling of the friction interface, allowing the combination of several properties, including anti-wear (AW) and extreme pressure (EP) additives, as well as friction modifiers (FM). Owing to their low melting point and high chemical reactivity, NPs can deposit on microdefects of friction surfaces and, to some extent, play the role of "self-repairing" [19]. In addition, NPs have higher thermal conductivity than the base fluid, which facilitates the release of the heat generated by friction and contributes to the stability of the tribo-pairs. An essential advantage of nanolubricants is that they do not require triboactive elements such as phosphorus and sulfur to improve the tribological properties of the base oil, exhibiting excellent friction and wear reduction characteristics. NPs are of considerable interest for improving the properties of biodegradable lubricants. Finally, most NPs are environmentally friendly, as they minimize the use of hazardous materials and additives [16, 20], which is useful for environmental and economic sustainability. In addition, eco-friendly NPs may also facilitate the reduction of energy consumption in production processes, thus leading to a reduction of the carbon footprint. Nanolubricants meet the requirements of green tribology, which is a new area for a large number of tribologists [21, 22].

To date, a large number of nanomaterials used as additives to lubricating oils, in particular, carbon materials [23, 24], carbon nanotubes [25], graphene oxide [26-28], boron nitrides [29, 30], and silicon oxide [31], have been obtained. However, the most extensive studies have been carried out with metal-containing nanomaterials, whose NPs contain, e.g., metals, their oxides, and sulfides [32-34]. Importantly, some of the nanomaterials studied are commercial products [35-39],

* Corresponding author: Igor E. UFLYAND, E-mail: ieuflyand@sfedu.ru 
but most studies are concerned with self-made NPs. Various chemical and physical methods are used to obtain NPs [40-44], and they continue to improve. An interesting example is the use of self-propagating high-temperature synthesis for the production of various tribological nanomaterials [45].

It is well known that lubrication can be divided into three different regimes: boundary lubrication, mixed lubrication, and elastohydrodynamic/hydrodynamic lubrication (Fig. 1) [9, 46, 47].

Among them, friction and wear are particularly high in boundary and mixed lubrication, which leads to high machine wear and energy loss [48, 49]. Consequently, lubricant additives are highly important in boundary lubrication owing to the higher coefficient of friction (COF) [16, 50]. Actual problems of reducing friction and wear require an adaptable lubricant for various operating conditions. Accordingly, a large amount of research has focused on the concept of nanolubrication in internal combustion engines as the main strategy for reducing COF and the wear of contact surfaces, which ultimately leads to improved tribological characteristics [51].

To date, despite a significant number of experimental studies on NPs as lubricant additives, several aspects of their tribological behavior have not yet been fully understood. This review will summarize the latest advances in the field of nanolubricants based on metal-containing nanomaterials over the past few years. The rapid growth of this area makes this review timely. No exhaustive analysis of the entire array of current experimental data will be attempted; rather, the focus will primarily be on the composition, the factors influencing the tribological characteristics,
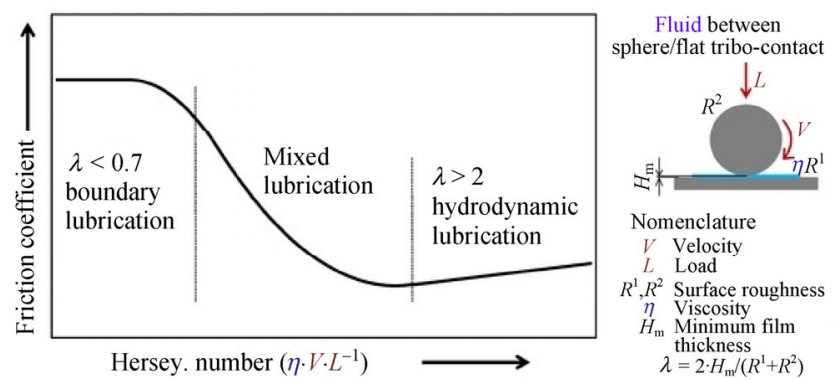

Fig. 1 Stribeck curve and lubrication regime [9]. $H_{\mathrm{m}}$ (minimum thickness of liquid film developed from the base fluid between the surfaces, clearance of the surfaces) could be calculated from the operating parameters (load and velocity) and material parameters (elastic modulus and pressure-viscosity relations). and the mechanisms of friction of metal-containing nanolubricants.

\section{Composition of metal-containing nanolubricants}

In most of the studies carried out, it is noted that the addition of NPs to the lubricant can increase its tribological characteristics, which largely depend on the composition of the lubricant [39, 47, 52-58]. According to the data in Ref. [15], metal-containing nanomaterials account for $72 \%$ of the nanolubricants studied.

\subsection{Metals}

Metallic NPs have unique chemical and physical properties as lubricant additives [59-71]. Nano-metals with low shear stress, high extension, and low melting point have been used as FMs owing to their excellent friction-reducing, anti-wear, and self-repairing ability.

Among metallic NPs, Cu-containing nanolubricants have received particular attention owing to their remarkable properties [59-71]. Copper NPs usually have small particle size, low melting point, and the desired ductility; therefore, they are well perceived as an excellent AW and EP agent in comparison with similar products $[72,73]$. Copper NPs as an additive can significantly improve the tribological properties of lubricants, which allows the necessary lubrication of equipment. A typical example is the use of two commercially available base oils with synthetic engine oil SAE 5W40 grades dispersed with $0.2 \mathrm{wt} \% \mathrm{Cu}$ NPs [74]. A significant reduction in friction and wear on the order of less than $13 \%$ was observed, and was tribological performance of base oils. Cu nanolubricants form boundary films on friction surfaces, thus increasing tribo efficiency by reducing friction and wear.

In another interesting example, the tribological properties of nanolubricants based on $\mathrm{Fe}, \mathrm{Cu}$, and Co NPs, which were added individually and in pairs into mineral oil, were estimated [59]. Cu-containing nanolubricants significantly reduced friction and wear compared to other NPs when added individually. In particular, the presence of $\mathrm{Cu}, \mathrm{Fe}$, and $\mathrm{Co}$ NPs reduced friction by $49 \%, 39 \%$, and $20 \%$, respectively, compared to lubricants without additives. When they 
were added in pairs, nanolubricants containing $\mathrm{Fe}-\mathrm{Cu}$ and $\mathrm{Co}-\mathrm{Cu}$ exhibited a decrease in friction of up to $53 \%$, whereas $\mathrm{Fe}-\mathrm{Co}$ resulted in a $36 \%$ decrease.

It is of interest to use Ag and Au NPs as nanoadditives to lubricant compositions [75, 76]. In particular, the use of Ag NPs modified by thiolated ligands, 4-(tert-butyl)benzylthiol, and dodecanethiol in the base oil reduced friction by up to $35 \%$ and wear by up to $85 \%$ in boundary lubrication [77].

It is also interesting to study the friction-reducing and anti-wear behavior of multialkylated cyclopentanes oil with Mo and W NPs as additives under vacuum conditions $\left(\sim 10^{-4} \mathrm{~Pa}\right)[78,79]$. The oil exhibited transient high friction in vacuum, resulting into strong adhesion wear of the steel friction pairs, which can be effectively eliminated by Mo and $\mathrm{W}$ nano-additives.

The lubrication mechanisms of metal NPs can be grouped as follows: (a) Tribo-films or adsorption films are formed. These films change the surface properties and separate the two friction surfaces, thus giving promising tribological characteristics. (b) The added NPs are rolled within two sliding surfaces, resulting in a reduction in friction and wear. (c) NPs are compacted on the wear track owing to the heat and pressure generated during the friction process. This phenomenon is called sintering or repair effect.

An overview of the typical representatives of metallic NPs used as lubricant additives is given in Table 1.

Table 1 Metallic NPs as lubricant additives.

\begin{tabular}{|c|c|c|c|c|c|c|c|c|c|c|}
\hline \multicolumn{4}{|c|}{ Nanolubricant details } & \multirow[b]{2}{*}{ Tribometer } & \multicolumn{3}{|c|}{ Conditions } & \multicolumn{2}{|c|}{ Tribological test results } & \multirow[b]{2}{*}{ Ref } \\
\hline $\begin{array}{l}\text { Nano- } \\
\text { materials }\end{array}$ & Grain size & Concentration & Base oil & & Load & Speed & $\begin{array}{c}\text { Tem- } \\
\text { perature }\end{array}$ & $\begin{array}{c}\text { Wear } \\
\text { reduction, \% }\end{array}$ & $\begin{array}{l}\text { Friction } \\
\text { reduction, \% }\end{array}$ & \\
\hline \multirow{2}{*}{$\mathrm{Bi}$} & \multirow{2}{*}{$7-65 \mathrm{~nm}$} & $900 \mathrm{mg} / \mathrm{L}$ & $\begin{array}{l}\text { Light base } \\
\text { oil }\end{array}$ & Four-ball tester & \multirow{2}{*}{$392 \mathrm{~N}$} & \multirow{2}{*}{$1,200 \mathrm{rpm}$} & \multirow{2}{*}{$75^{\circ} \mathrm{C}$} & \multirow{2}{*}{ - } & $\begin{array}{c}\text { From } 0.091 \text { to } \\
0.052\end{array}$ & \multirow{2}{*}{$\begin{array}{r}{[80} \\
81]\end{array}$} \\
\hline & & $310 \mathrm{mg} / \mathrm{L}$ & $\begin{array}{l}\text { Heavy } \\
\text { base oil }\end{array}$ & $\begin{array}{l}\text { Pin-on-disc } \\
\text { setup }\end{array}$ & & & & & $\begin{array}{c}\text { From } 0.074 \text { to } \\
0.047\end{array}$ & \\
\hline \multirow{6}{*}{$\mathrm{Cu}$} & & $3 \mathrm{wt} \%$ & $\begin{array}{c}\text { Chevron Taro } \\
30 \text { DP } 40 \text { and } \\
\text { Teboil Ward }\end{array}$ & $\begin{array}{l}\text { Pin-on-disc } \\
\text { setup }\end{array}$ & $\begin{array}{c}0.1- \\
180 \mathrm{mN}\end{array}$ & $0.02 \mathrm{~mm} / \mathrm{s}$ & $25^{\circ} \mathrm{C}$ & $\begin{array}{c}0.023- \\
0.018 \mathrm{mg}\end{array}$ & $\begin{array}{c}\text { From } 0.15 \text { to } \\
0.11\end{array}$ & {$[82]$} \\
\hline & $45 \mathrm{~nm}$ & $0.20 \mathrm{wt} \%$ & $\begin{array}{l}\text { SAE grade } \\
5 \mathrm{~W}-40\end{array}$ & $\begin{array}{l}\text { Four ball } \\
\text { tribotester }\end{array}$ & $40 \mathrm{~N}$ & $1,200 \mathrm{rpm}$ & $75^{\circ} \mathrm{C}$ & $\begin{array}{c}2.94- \\
13.30 \%\end{array}$ & $\begin{array}{c}\text { From } 2.58 \text { to } \\
4.76 \%\end{array}$ & {$[64]$} \\
\hline & & $0.15 \mathrm{wt} \%$ & $\begin{array}{l}\text { Mineral oil } \\
\text { SN } 650\end{array}$ & $\begin{array}{c}\text { Optimol SRV } 4 \\
\text { tester }\end{array}$ & $400 \mathrm{~N}$ & $200 \mathrm{rpm}$ & $\mathrm{rt}$ & $32 \%$ & $34 \%$ & {$[83]$} \\
\hline & $2-6 \mathrm{~nm}$ & $1.5 \mathrm{wt} \%$ & Paraffin oil & $\begin{array}{l}\text { Four-ball } \\
\text { machine }\end{array}$ & $300 \mathrm{~N}$ & $1,450 \mathrm{rpm}$ & & $\begin{array}{c}0.665- \\
0.460 \mathrm{~mm}\end{array}$ & & [84] \\
\hline & $10-60 \mathrm{~nm}$ & $0.02 \mathrm{wt} \%$ & Paraffin oil & $\begin{array}{l}\text { Four-ball } \\
\text { machine }\end{array}$ & $300 \mathrm{~N}$ & $1,450 \mathrm{rpm}$ & $20{ }^{\circ} \mathrm{C}$ & & $26 \%$ & {$[85]$} \\
\hline & $2-5 \mathrm{~nm}$ & & Paraffin oil & $\begin{array}{l}\text { Four-ball } \\
\text { friction and } \\
\text { wear tester }\end{array}$ & & & & \multicolumn{2}{|c|}{$\begin{array}{c}\text { Reduced the COF of } \\
\text { steel pair }\end{array}$} & {$[86]$} \\
\hline $\mathrm{Fe}, \mathrm{Cu}, \mathrm{Co}$ & & $0.5 \mathrm{wt} \%$ & $\begin{array}{c}\text { SAE } 10 \\
\text { mineral oil }\end{array}$ & $\begin{array}{l}\text { Four ball } \\
\text { tribotester }\end{array}$ & $150 \mathrm{~N}$ & $1,420 \mathrm{~min}^{-1}$ & & \multicolumn{2}{|c|}{$\begin{array}{c}\text { Up to } 20 \%(\mathrm{Co}) \\
39 \%(\mathrm{Fe}), 49 \%(\mathrm{Cu})\end{array}$} & [59] \\
\hline $\mathrm{Ni}$ & $\begin{array}{c}7.5-13.5 \mathrm{~nm} \\
\text { and } \\
28.5 \mathrm{~nm}\end{array}$ & $0.05 \mathrm{wt} \%$ & PAO & $\begin{array}{l}\text { Four-ball } \\
\text { friction and } \\
\text { wear tester }\end{array}$ & $300 \mathrm{~N}$ & $1,450 \mathrm{rpm}$ & & $0.47-0.54$ & $0.78-0.68$ & $\begin{array}{l}{[87,} \\
88]\end{array}$ \\
\hline $\mathrm{Pd}$ & $2 \mathrm{~nm}$ & $2 w t \%$ & Paraffin & $\begin{array}{l}\text { Ball-on-disk } \\
\text { tribometer }\end{array}$ & $1-20 \mathrm{~N}$ & $10 \mathrm{~cm} / \mathrm{s}$ & & \multicolumn{2}{|c|}{ Reduced COF } & [89] \\
\hline $\begin{array}{c}\mathrm{Sn} \\
\text { and } \mathrm{Fe}\end{array}$ & $\begin{array}{l}30-60 \mathrm{~nm} \\
\text { and } \\
20-70 \mathrm{~nm}\end{array}$ & $1 \mathrm{wt} \%$ & $\begin{array}{l}\text { Multialkylated } \\
\text { cyclopentanes }\end{array}$ & $\begin{array}{l}\text { Four-ball } \\
\text { tribometer }\end{array}$ & $300 \mathrm{~N}$ & $1,450 \mathrm{rpm}$ & $25^{\circ} \mathrm{C}$ & \multicolumn{2}{|c|}{$\begin{array}{l}\text { Sn is effective on friction } \\
\text { reduction; Fe is effective on } \\
\text { anti-wear performance, low } \\
\text { COF around } 0.1\end{array}$} & [90] \\
\hline $\begin{array}{l}\text { Mo } \\
\text { W }\end{array}$ & $\begin{array}{l}20-50 \mathrm{~nm} \\
30-60 \mathrm{~nm}\end{array}$ & $\begin{array}{c}0.1-0.5 \mathrm{wt} \% \\
1.0 \mathrm{wt} \%\end{array}$ & $\begin{array}{l}\text { Multialkylated } \\
\text { cyclopentanes }\end{array}$ & $\begin{array}{c}\text { Four ball } \\
\text { fatigue tester }\end{array}$ & $294 N$ & $1,450 \mathrm{rpm}$ & $\mathrm{rt}$ & \multicolumn{2}{|c|}{$\begin{array}{c}\text { Low COF of } \\
\text { approximately } 0.1\end{array}$} & [78] \\
\hline
\end{tabular}




\subsection{Metal oxides}

Various metal oxides are used as lubricant additives, including $\mathrm{TiO}_{2}, \mathrm{CuO}, \mathrm{Fe}_{3} \mathrm{O}_{4}, \mathrm{ZnO}, \mathrm{Co}_{3} \mathrm{O}_{4}$, and $\mathrm{Al}_{2} \mathrm{O}_{3}$ $[34,55,91]$. Their lubrication mechanisms are analogous to those of metal-containing nanomaterials, including the formation of tribo-film or adsorption film, the rolling effect, and the sintering or repair effect. A typical example is the use of spherical $\mathrm{CuO}$ and $\mathrm{TiO}_{2}$ NPs as lubricant additives, exhibiting good friction reduction and anti-wear behavior, particularly for $\mathrm{CuO}$ [56]. The friction reduction can be explained by the effect of viscosity at low temperature and the rolling effect at high temperature, and the anti-wear mechanism is associated with the deposition of $\mathrm{CuO}$ NPs on the friction surface, which can reduce shearing stress and improve tribological properties.

Several studies are devoted to the use of $\mathrm{TiO}_{2} \mathrm{NPs}$ as lubricant additives [92-96]. In particular, a sample of palm oil biolubricant with $0.1 \mathrm{wt} \%$ of a $\mathrm{TiO}_{2}$ nanoadditive had the lowest $\mathrm{COF}$ and wear scar diameter.

The use of oxide NPs $(\mathrm{ZnO}$ and $\mathrm{CuO})$ as lubricant additives should be noted [97-104]. For example, biolubricants using vegetable oils (soybean and sunflower) with $\mathrm{ZnO}$ and $\mathrm{CuO}$ NPs as additives are biodegradable and have better performance in boundary lubrication.

Magnetic $\mathrm{Fe}_{3} \mathrm{O}_{4}$ NPs with an average particle diameter of $11.7 \mathrm{~nm}$ were dispersed in alpha-olefin hydrocarbon synthetic lubricating oil with a solid weight fraction of 0 to $10 \mathrm{wt} \%$ [105, 106]. This resulted in a reduction in $\mathrm{COF}$ and the diameter of the wear scar by $45 \%$ and $30 \%$, respectively, at the optimal value, i.e., $4 \mathrm{wt} \%$ of the concentration of the NPs. The rolling mechanism is responsible for the reduction of COF, whereas the magnetic NPs act as the spacer between the asperities and reduce the diameter of the wear scar.

It is of interest to use copper [107-111] and cerium oxide NPs [112] as lubricant additives to improve tribological characteristics. A nanolubricant based on palm oil with the addition of copper oxide NPs exhibited $20.12 \%$ and $8.73 \%$ lower COF compared to mineral-based engine oil (SAE 40) and palm kernel oil, respectively [113]. However, it represented 10.13\% and $1.74 \%$ higher wear scar diameter than SAE 40 and palm kernel oil, respectively.
The typical representatives of nanolubricants based on metal oxide NPs are listed in Table 2.

\subsection{Metal sulfides}

Although $\mathrm{MoS}_{2}$ has been widely used as an important lubricant additive for a long time, it has been demonstrated that $\mathrm{MoS}_{2}$ NPs as FMs in liquid lubricants are superior to $\mathrm{MoS}_{2}$ microparticles, owing to the chemical stability of the layer-closed spherical structure of NPs. Both $\mathrm{MoS}_{2}$ and $\mathrm{WS}_{2}$ NPs, which are layered compounds with a hollow polyhedral structure known as fullerene-like NPs (IF-NPs), have proven to be good FMs when dispersed in lubricants [130].

It is of interest to study the anti-friction behavior of FeS NPs with a size ranging from 20 to $200 \mathrm{~nm}$ as a lubricating oil additive in engine oil [131]. COF decreases remarkably with the addition of these NPs; furthermore, a persistent antifriction effect under dry condition is observed. It is important that the diffusion of $S$ atoms in the near-surface region forms a sulfur diffusing area, resulting in a durable friction-reduction behavior on the friction pair.

Examples of the use of metal sulfide NPs in nanolubricants are presented in Table 3.

\subsection{Nanocomposites}

Owing to the synergetic effect of more than one NP type, composites usually exhibit superior performance compared to individual NPs [136-138]. Thus, $\mathrm{Cu} / \mathrm{CeO}_{2}$, $\mathrm{Al}_{2} \mathrm{O}_{3} / \mathrm{SiO}_{2}, \mathrm{ZrO}_{2} / \mathrm{SiO}_{2}$, and ( $\left.\mathrm{Zn}-\mathrm{Ni}\right) /$ nano $\mathrm{Al}_{2} \mathrm{O}_{3}$ composite NPs were used as energy-efficient lubricant additives [139]. Lubricant additives such as nano-Ag/ multi-wall carbon nanotubes [140] and silver NPdecorated graphene [141] should be mentioned.

It is of interest to study the tribological properties of composite nanomaterials based on zinc oxide NPs and nanolamellar tungsten and molybdenum disulfide $[142,143]$. According to tribological measurements, the addition of $\mathrm{ZnO}$ NPs did not significantly alter the COF of nanolamellar metal disulfides at $25^{\circ} \mathrm{C}$ in air, whereas it positively affected wear resistance at $400{ }^{\circ} \mathrm{C}$.

$\mathrm{Cu}$ NPs and Ag NPs were used as metal cladding modifiers of nanolamellar $\mathrm{MoS}_{2}[144,145]$ and $\mathrm{WS}_{2}$ [137] particles. It was demonstrated that such nanocomposite lubricants changed the COF of the original 
Table 2 Metal oxide NPs as lubricant additives.

\begin{tabular}{|c|c|c|c|c|c|c|c|c|c|c|}
\hline \multicolumn{4}{|c|}{ Nanolubricant details } & \multirow[b]{2}{*}{ Tribometer } & \multicolumn{3}{|c|}{ Conditions } & \multicolumn{2}{|c|}{ Tribological test results } & \multirow[b]{2}{*}{ Ref. } \\
\hline $\begin{array}{l}\text { Nano- } \\
\text { materials }\end{array}$ & Grain size & $\begin{array}{l}\text { Concen- } \\
\text { tration }\end{array}$ & Base oil & & Load & Speed & $\begin{array}{l}\text { Tem- } \\
\text { perature }\end{array}$ & $\begin{array}{l}\text { Wear } \\
\text { reduction, \% }\end{array}$ & $\begin{array}{c}\text { Friction reduction, } \\
\%\end{array}$ & \\
\hline \multirow{10}{*}{$\mathrm{TiO}_{2}$} & $20-25 \mathrm{~nm}$ & $0.25 \mathrm{wt} \%$ & Mineral oil & $\begin{array}{l}\text { Four ball } \\
\text { tribo-tester }\end{array}$ & $14.7 \mathrm{~N}$ & $0.05 \mathrm{~m} / \mathrm{s}$ & $\mathrm{rt}$ & - & $21 \%$ & {$[55]$} \\
\hline & $30 \mathrm{~nm}$ & $2 \mathrm{wt} \%$ & $\begin{array}{l}\text { Oil-in-water } \\
\text { lubricant }\end{array}$ & $\begin{array}{l}\text { Rtec MFT- } \\
5,000 \text { multi- } \\
\text { functional high } \\
\text { temperature } \\
\text { tribometer }\end{array}$ & $50 \mathrm{~N}$ & $\begin{array}{c}10- \\
70 \mathrm{~mm} / \mathrm{s}\end{array}$ & $80{ }^{\circ} \mathrm{C}$ & - & $16.3 \%$ & {$[114]$} \\
\hline & $30 \mathrm{~nm}$ & $\begin{array}{l}0,0.5,2,4 \\
\text { and } 6 \mathrm{wt} \%\end{array}$ & $\begin{array}{c}\text { Oil-in-water } \\
\text { lubricant }\end{array}$ & $\begin{array}{l}\text { Ball-on-disk } \\
\text { tester }\end{array}$ & $50 \mathrm{~N}$ & $50 \mathrm{~mm} / \mathrm{s}$ & $80{ }^{\circ} \mathrm{C}$ & Wear was sig & gnificantly reduced & {$[115]$} \\
\hline & $20 \mathrm{~nm}$ & $4 \mathrm{wt} \%$ & Water based oil & $\begin{array}{l}\text { Ball-on-disk } \\
\text { tribometer }\end{array}$ & $5 \mathrm{~N}$ & $50 \mathrm{~mm} / \mathrm{s}$ & $\mathrm{rt}$ & $34 \%$ & $20 \%$ & {$[116]$} \\
\hline & $20 \mathrm{~nm}$ & $\begin{array}{c}0.1- \\
1.6 \mathrm{wt} \%\end{array}$ & $\begin{array}{l}\text { Water-based } \\
\text { cutting fluids }\end{array}$ & $\begin{array}{l}\text { Four-ball } \\
\text { tribotester }\end{array}$ & $147 \mathrm{~N}$ & $1,440 \mathrm{rpm}$ & & $34.8 \%$ & from 0.17 to 0.04 & {$[117]$} \\
\hline & $<100 \mathrm{~nm}$ & $\begin{array}{c}\text { volume } \\
\text { fractions of } \\
0.0001 \text { to } \\
0.005\end{array}$ & SAE30 engine oil & & & & & & $40 \%$ & [118] \\
\hline & & $0.1 \mathrm{wt} \%$ & $\begin{array}{l}\text { Biolubricant palm } \\
\text { oil-based trimethy- } \\
\text { lolpropane ester }\end{array}$ & $\begin{array}{l}\text { Four-ball } \\
\text { machine } \\
\text { tribotester }\end{array}$ & $160 \mathrm{~kg}$ & $1,200 \mathrm{rpm}$ & $\mathrm{rt}$ & $11 \%$ & $15 \%$ & [119] \\
\hline & $10-25 \mathrm{~nm}$ & $1.5 \mathrm{wt} \%$ & $\begin{array}{l}\text { multi-grade engine } \\
\text { oil Castrol Active } \\
\text { 4T SAE 20W } 40\end{array}$ & $\begin{array}{l}\text { Pin-on-disc } \\
\text { tribometer }\end{array}$ & $\begin{array}{c}40,60 \\
\text { and } 90 \mathrm{~N}\end{array}$ & $\begin{array}{l}0.5,1.0 \text { and } \\
1.5 \mathrm{~m} / \mathrm{s}\end{array}$ & & \multicolumn{2}{|c|}{ Wear was significantly reduced } & {$[120]$} \\
\hline & $\begin{array}{c}50- \\
100 \mathrm{~nm}\end{array}$ & $\begin{array}{l}0.1,0.2 \\
0.3,0.4 \\
0.5 \mathrm{wt} \%\end{array}$ & $\begin{array}{c}\text { API-1509 } \\
\text { base oil }\end{array}$ & $\begin{array}{l}\text { Four-ball } \\
\text { tribometer and } \\
\text { ball-on-disk } \\
\text { tribometer }\end{array}$ & $100 \mathrm{~N}$ & & $75^{\circ} \mathrm{C}$ & \multicolumn{2}{|c|}{$\begin{array}{l}\text { Friction and wear reduction } \\
\text { compared to the base oil }\end{array}$} & {$[121]$} \\
\hline & & $\begin{array}{l}\text { from } 0.1 \text { to } \\
2.0 \mathrm{wt} \%\end{array}$ & Vaseline oil & $\begin{array}{l}\text { Four-ball } \\
\text { tribometer }\end{array}$ & $200 \mathrm{~N}$ & $1,460 \mathrm{rpm}$ & & & $29.4 \%$ & {$[122]$} \\
\hline $\mathrm{SnO}_{2}$ & & $\begin{array}{l}\text { from } 0.5 \text { to } \\
5 \mathrm{wt} \%\end{array}$ & PAO & $\begin{array}{l}\text { Bruker universal } \\
\text { mechanical } \\
\text { tribometer }\end{array}$ & $25 \mathrm{~N}$ & & $\mathrm{rt}$ & $43.7 \%$ & $65.4 \%$ & {$[123]$} \\
\hline \multirow[b]{2}{*}{$\mathrm{Al}_{2} \mathrm{O}_{3}$} & $78 \mathrm{~nm}$ & $0.1 \mathrm{wt} \%$ & Base oil & $\begin{array}{l}\text { Four-ball } \\
\text { tribometer }\end{array}$ & $200 \mathrm{~N}$ & $1,200 \mathrm{rpm}$ & $75^{\circ} \mathrm{C}$ & $41 \%$ & $17-24 \%$ & {$[58]$} \\
\hline & $40-80 \mathrm{~nm}$ & $0-1 \mathrm{wt} \%$ & $\begin{array}{l}\text { SAE20W40 } \\
\text { lubricating oil }\end{array}$ & $\begin{array}{l}\text { Pin-on-disc } \\
\text { tribotester }\end{array}$ & $160 \mathrm{~N}$ & $1,200 \mathrm{rpm}$ & & \multicolumn{2}{|c|}{$\begin{array}{l}\text { The minimum COF was obtained } \\
\text { with } 0.8 \mathrm{wt} \% \text { of medium NPs size } \\
60 \mathrm{~nm}\end{array}$} & {$[124]$} \\
\hline $\begin{array}{l}\mathrm{Al}_{2} \mathrm{O}_{3} \text { and } \\
\quad \mathrm{CuO}\end{array}$ & $<50 \mathrm{~nm}$ & $\begin{array}{l}0.5,1.0 \\
\text { and } \\
2.0 \mathrm{wt}^{\mathrm{O}} \%\end{array}$ & $\begin{array}{l}\text { SAE 75W85 } \\
\text { and PAO8 }\end{array}$ & $\begin{array}{l}\text { Optimol SRV } 4 \\
\text { tester }\end{array}$ & $\begin{array}{c}\text { from } \\
0 \text { to } \\
7,200 \mathrm{~N}\end{array}$ & $500 \mathrm{rpm}$ & $25^{\circ} \mathrm{C}$ & & $\begin{array}{l}\text { up to } 18 \% \text { and } \\
14 \%\end{array}$ & {$[125]$} \\
\hline $\begin{array}{l}\mathrm{Al}_{2} \mathrm{O}_{3} \text { and } \\
\mathrm{TiO}_{2}\end{array}$ & $\begin{array}{l}8-12 \mathrm{~nm} \\
10 \mathrm{~nm}\end{array}$ & $0.25 \mathrm{wt} \%$ & $5 W-30$ & $\begin{array}{l}\text { Piston ring/ } \\
\text { cylinder liner } \\
\text { contact tester }\end{array}$ & $\begin{array}{c}120- \\
250 \mathrm{~N}\end{array}$ & $0.5 \mathrm{~m} / \mathrm{s}$ & $100{ }^{\circ} \mathrm{C}$ & $\begin{array}{l}21 \% \\
29 \%\end{array}$ & $\begin{array}{l}45 \% \\
50 \%\end{array}$ & {$[47]$} \\
\hline $\begin{array}{l}\mathrm{ZnO} \text { and } \\
\mathrm{CuO}\end{array}$ & $\begin{array}{l}11.71 \mathrm{~nm} \\
14.35 \mathrm{~nm}\end{array}$ & $0.5 \mathrm{wt} \%$ & $\begin{array}{l}\text { Mineral, PAO, } \\
\text { sunflower, } \\
\text { soybean }\end{array}$ & $\begin{array}{l}\text { High frequency } \\
\text { reciprocating } \\
\text { test rig }\end{array}$ & $10 \mathrm{~N}$ & & $50{ }^{\circ} \mathrm{C}$ & \multicolumn{2}{|c|}{$\begin{array}{l}\mathrm{ZnO} \text { is effective with mineral oil. } \\
\mathrm{CuO} \text { is effective with synthetic oil. }\end{array}$} & [126] \\
\hline \multirow{2}{*}{$\mathrm{ZnO}$} & & $0.5 \mathrm{wt} \%$ & 60SN base oil & $\begin{array}{l}\text { Four-ball } \\
\text { machine }\end{array}$ & & & & $9.9 \%$ & $31.2 \%$ & [127] \\
\hline & $4.04 \mathrm{~nm}$ & $1.20 \mathrm{wt} \%$ & $\begin{array}{l}\text { Lubricant } \\
\text { base oils }\end{array}$ & $\begin{array}{l}\text { Pin-on-disc } \\
\text { tribotester }\end{array}$ & $392 \mathrm{~N}$ & $1,200 \mathrm{rpm}$ & $75^{\circ} \mathrm{C}$ & $31.2 \%$ & $9.9 \%$ & [128] \\
\hline
\end{tabular}




\begin{tabular}{|c|c|c|c|c|c|c|c|c|c|c|}
\hline \multicolumn{11}{|c|}{ (Continued) } \\
\hline \multicolumn{4}{|c|}{ Nanolubricant details } & \multirow[b]{2}{*}{ Tribometer } & \multicolumn{3}{|c|}{ Conditions } & \multicolumn{2}{|c|}{ Tribological test results } & \multirow[b]{2}{*}{ Ref. } \\
\hline $\begin{array}{l}\text { Nano- } \\
\text { materials }\end{array}$ & Grain size & $\begin{array}{l}\text { Concen- } \\
\text { tration }\end{array}$ & Base oil & & Load & Speed & $\begin{array}{l}\text { Tem- } \\
\text { perature }\end{array}$ & $\begin{array}{c}\text { Wear } \\
\text { reduction, \% }\end{array}$ & $\begin{array}{l}\text { Friction } \\
\text { reduction, \% }\end{array}$ & \\
\hline $\mathrm{ZrO}_{2}$ & $<100 \mathrm{~nm}$ & $0.5 \mathrm{wt} \%$ & $20^{\#}$ machine oil & Four-ball tester & $392 \mathrm{~N}$ & $1,450 \mathrm{rpm}$ & $\mathrm{rt}$ & $3.98 \%$ & $5.36 \%$ & [129] \\
\hline $\begin{array}{c}\mathrm{Fe}_{3} \mathrm{O}_{4} \\
\text { magnetic } \\
\text { NPs }\end{array}$ & $11.7 \mathrm{~nm}$ & $4 \mathrm{wt} \%$ & $\begin{array}{l}\text { alpha olefin } \\
\text { hydrocarbon } \\
\text { synthetic } \\
\text { lubricating oil }\end{array}$ & Four-ball tester & $392 \mathrm{~N}$ & $\begin{array}{l}1,200 \pm \\
20 \mathrm{rpm}\end{array}$ & $75^{\circ} \mathrm{C}$ & $30 \%$ & $45 \%$ & [101] \\
\hline $\mathrm{Fe}_{3} \mathrm{O}_{4}$ & $\begin{array}{c}45.8- \\
50.1 \mathrm{~nm}\end{array}$ & $1.5 \mathrm{wt} \%$ & \#40 engine oil & Four-ball tester & $400 \mathrm{~N}$ & $1,450 \mathrm{rpm}$ & & $\begin{array}{r}\text { COF could be } \\
47.96 \% \text {, and } 34 \\
\text { could be red } \\
11.17 \% \text {, and } \\
\text { containing } \\
\text { hexagonal, octa } \\
\text { morphologi }\end{array}$ & $\begin{array}{l}\text { duced by } 58.16 \% \text {, } \\
69 \% \text {, and the wear } \\
\text { ced by } 13.87 \% \text {, } \\
.18 \% \text { for the oils } \\
{ }_{3} \mathrm{O}_{4} \mathrm{NPs} \text { with } \\
\text { edral, and irregular } \\
\text {, respectively. }\end{array}$ & [105] \\
\hline
\end{tabular}

Table 3 Metal sulfide NPs as lubricant additives.

\begin{tabular}{|c|c|c|c|c|c|c|c|c|c|c|}
\hline \multicolumn{4}{|c|}{ Nanolubricant details } & \multicolumn{2}{|l|}{ Tribometer } & \multicolumn{2}{|l|}{ Conditions } & \multicolumn{2}{|c|}{ Tribological test results } & \multirow[b]{2}{*}{ Ref. } \\
\hline $\begin{array}{l}\text { Nano- } \\
\text { materials }\end{array}$ & Grain size & Concentration & Base oil & & Load & Speed & $\begin{array}{l}\text { Tem- } \\
\text { perature }\end{array}$ & $\begin{array}{c}\text { Wear } \\
\text { reduction, \% }\end{array}$ & $\begin{array}{l}\text { Friction } \\
\text { reduction, \% }\end{array}$ & \\
\hline $\mathrm{FeS}$ & $20-200 \mathrm{~nm}$ & 0 to $2 \mathrm{wt} \%$ & $\begin{array}{l}\text { Commercial API } \\
\text { SL/CF 10W-40 } \\
\text { engine oil }\end{array}$ & $\begin{array}{l}\text { Pin-on-disc } \\
\text { system }\end{array}$ & $\begin{array}{l}50 \text { or } \\
150 \mathrm{~N}\end{array}$ & $150 \mathrm{rpm}$ & & $\begin{array}{r}\text { COF decreas } \\
\text { under oil lubi } \\
\text { conc }\end{array}$ & $\begin{array}{l}\text { ses remarkably } \\
\text { rication and dry } \\
\text { ditions }\end{array}$ & [131] \\
\hline \multirow{5}{*}{$\mathrm{MoS}_{2}$} & $\begin{array}{l}350 \text { and } \\
150 \mathrm{~nm}\end{array}$ & $1 \mathrm{wt} \%$ & $\begin{array}{l}\text { Blend of PAO } 4 \\
\text { and PAO } 40\end{array}$ & $\begin{array}{c}\text { High } \\
\text { Frequency } \\
\text { Reciprocating } \\
\text { Rig }\end{array}$ & $10 \mathrm{~N}$ & & $80^{\circ} \mathrm{C}$ & & $\begin{array}{l}\text { From } 0.20 \text { to } \\
0.06\end{array}$ & [132] \\
\hline & $50-100 \mathrm{~nm}$ & $\begin{array}{c}2.0,1.5,1.0, \\
0.5,0.25 \text {, and } \\
0 \mathrm{wt} \%\end{array}$ & Dioctyl sebacate & $\begin{array}{c}\text { High-frequency } \\
\text { reciprocating } \\
\text { ball-on-disc } \\
\text { tribometer }\end{array}$ & $7.84 \mathrm{~N}$ & $0.1 \mathrm{~m} / \mathrm{s}$ & $60{ }^{\circ} \mathrm{C}$ & $35 \%$ & $\sim 37 \%$ & [133] \\
\hline & $\begin{array}{l}\text { Diameters below } \\
100 \mathrm{~nm} \text { and } \\
\text { lengths up to } \\
20 \mu \mathrm{m}\end{array}$ & $2 \mathrm{wt} \%$ & $\mathrm{PAO}$ & $\begin{array}{l}\text { Ball-on-disc } \\
\text { tribometer }\end{array}$ & $35 \mathrm{~N}$ & $\begin{array}{l}\text { from } 3.2 \text { to } \\
0.002 \mathrm{~m} / \mathrm{s}\end{array}$ & $\mathrm{rt}$ & & From 30 to $50 \%$ & [134] \\
\hline & $\begin{array}{l}\text { Average diameter } \\
\sim 50 \mathrm{~nm} \text {; single } \\
\text { layer thickness } \\
\sim 3 \mathrm{~nm}\end{array}$ & $\approx 1 \mathrm{wt} \%$ & SE $15 W-40$ & $\begin{array}{l}\text { Disc-on- } \\
\text { disc frictional } \\
\text { testing } \\
\text { machine }\end{array}$ & $1,500 \mathrm{~N}$ & $500 \mathrm{rpm}$ & $\mathrm{rt}$ & \multicolumn{2}{|c|}{$\begin{array}{l}\text { Enhance significantly } \\
\text { tribological performance }\end{array}$} & [135] \\
\hline & $90 \mathrm{~nm}$ & $\begin{array}{l}0.53 \mathrm{wt} \% \\
0.58 \mathrm{wt} \%\end{array}$ & $\begin{array}{c}\text { vegetable } \\
\text { (coconut) oil and } \\
\text { a mineral oil } \\
(500 \mathrm{~N} \text { base-oil) }\end{array}$ & $\begin{array}{l}\text { Pin-on-disc } \\
\text { tribometer } \\
\text { and a four- } \\
\text { ball tester }\end{array}$ & $\begin{array}{c}100- \\
200 \mathrm{~N}\end{array}$ & $\begin{array}{c}100- \\
300 \mathrm{rpm}\end{array}$ & $\begin{array}{c}30- \\
120^{\circ} \mathrm{C}\end{array}$ & \multicolumn{2}{|c|}{$\begin{array}{l}\text { Enhance significantly } \\
\text { tribological performance }\end{array}$} & [35] \\
\hline
\end{tabular}

lubricant and significantly improved its wear resistance.

In Ref. [138], the tribological behavior of decorative thin-film nanocomposities consisting of gold NPs dispersed in the $\mathrm{TiO}_{2}$ dielectric matrix was studied, and it was demonstrated that the clustering of gold, the increase in grain size, and the crystallization of the $\mathrm{TiO}_{2}$ dielectric matrix correlated with changes in tribological parameters.
Typical examples of lubricants based on nanocomposities are given in Table 4.

\subsection{Rare-earth compounds}

Among the rare-earth compounds studied, the most widely used elements were $\mathrm{La}$ and $\mathrm{Ce}$. Such compounds can be used either individually as lubricant additives or in other NPs such as $\mathrm{TiO}_{2}$. Their lubrication 
Table 4 Nanocomposite NPs as lubricant additives.

\begin{tabular}{|c|c|c|c|c|c|c|c|c|c|c|}
\hline \multicolumn{4}{|c|}{ Nanolubricant details } & \multirow[b]{2}{*}{ Tribometer } & \multicolumn{3}{|c|}{ Conditions } & \multicolumn{2}{|c|}{ Tribological test results } & \multirow[b]{2}{*}{ Ref. } \\
\hline Nanomaterials & Grain size & $\begin{array}{l}\text { Concen- } \\
\text { tration }\end{array}$ & Base oil & & Load & Speed & $\begin{array}{c}\text { Tem- } \\
\text { perature }\end{array}$ & $\begin{array}{c}\text { Wear } \\
\text { reduction, } \\
\%\end{array}$ & $\begin{array}{c}\text { Friction } \\
\text { reduction, } \\
\%\end{array}$ & \\
\hline $\mathrm{TiO}_{2} / \mathrm{SiO}_{2}$ & $50 \mathrm{~nm}$ & $0.75 \mathrm{wt} \%$ & Palm oil & $\begin{array}{l}\text { Four-ball } \\
\text { extreme } \\
\text { pressure and } \\
\text { piston ring- } \\
\text { cylinder liner } \\
\text { sliding } \\
\text { tribotesters }\end{array}$ & $160 \mathrm{~N}$ & $500 \mathrm{rpm}$ & $70{ }^{\circ} \mathrm{C}$ & $10.4 \%$ & $\sim 17-25 \%$ & {$[146]$} \\
\hline $\mathrm{Al}_{2} \mathrm{O}_{3} / \mathrm{SiO}_{2}$ & $70 \mathrm{~nm}$ & $\begin{array}{c}0.05,0.1 \\
0.5,1 \mathrm{wt}^{0} \%\end{array}$ & PAO 6 & $\begin{array}{l}\text { Pin-on-disk } \\
\text { tribometer }\end{array}$ & $\begin{array}{l}147 \text { and } \\
200 \mathrm{~N}\end{array}$ & $\begin{array}{l}1,200 \text { and } \\
1,450 \mathrm{rpm}\end{array}$ & $75^{\circ} \mathrm{C}$ & & $\sim 20 \%$ & $\begin{array}{c}{[139-} \\
141]\end{array}$ \\
\hline $\begin{array}{c}\text { Graphene and } \\
\mathrm{MoS}_{2}\end{array}$ & $\begin{array}{c}\text { Two-dimens } \\
\text { ional size is } \\
\sim 2 \mu \mathrm{m}\end{array}$ & $1.0 \mathrm{wt} \%$ & Hydraulic oil & $\begin{array}{l}\text { Ball-on-disk } \\
\text { reciprocating } \\
\text { friction tester }\end{array}$ & $3 \mathrm{~N}$ & $\begin{array}{c}1.2- \\
38.4 \mathrm{~mm} / \mathrm{s}\end{array}$ & $\begin{array}{c}25^{-} \\
125^{\circ} \mathrm{C}\end{array}$ & & $\begin{array}{c}\text { Up to as } \\
\text { low as } 0.04\end{array}$ & {$[147]$} \\
\hline Re:IF-MoS 2 & $100 \mathrm{~nm}$ & & PAO-6 & $\begin{array}{l}\text { Rotational disc } \\
\text { tribometer }\end{array}$ & $0-100 \mathrm{~N}$ & $3,000 \mathrm{rpm}$ & $\begin{array}{c}25,50 \text { and } \\
80{ }^{\circ} \mathrm{C}\end{array}$ & $\begin{array}{l}\text { The bes } \\
\text { boundary } \\
\text { elasto-hy } \\
\text { lubricati }\end{array}$ & $\begin{array}{l}\text { results in } \\
\text { mixed and } \\
\text { Irodynamic } \\
\text { on regimes }\end{array}$ & {$[130]$} \\
\hline $\mathrm{WS}_{2}-\mathrm{Cu}$ & $50-80 \mathrm{~nm}$ & & & $\begin{array}{l}\text { Ball-on-disk } \\
\text { High } \\
\text { Temperature } \\
\text { Tribometer }\end{array}$ & $\begin{array}{l}5 \text { and } \\
10 \mathrm{~N}\end{array}$ & $5 \mathrm{~cm} / \mathrm{s}$ & Rt & $\begin{array}{r}\text { Reduce } \\
\text { friction } \\
\text { using both } \\
\text { and nan } \\
\text { molybden }\end{array}$ & $\begin{array}{l}\text { vear of the } \\
\text { ody when } \\
\text { commercial } \\
\text { olamellar } \\
\text { im disulfide }\end{array}$ & {$[145]$} \\
\hline $\begin{array}{c}\mathrm{MoS}_{2}, \mathrm{WS}_{2} \text { and } \\
\mathrm{ZnO}\end{array}$ & & & & $\begin{array}{l}\text { Ball-on-disk } \\
\text { High } \\
\text { Temperature } \\
\text { Tribometer }\end{array}$ & & & $25^{\circ} \mathrm{C}$ & $\begin{array}{l}\mathrm{ZnO} \text { NPs } \\
\text { not practic }\end{array}$ & $\begin{array}{l}\text { additive did } \\
\text { ally change } \\
\text { OF }\end{array}$ & {$[143]$} \\
\hline $\mathrm{WS}_{2}$ and $\mathrm{ZnO}$ & $\begin{array}{c}50-150 \mathrm{~nm} \\
24 \mathrm{~nm}\end{array}$ & & & $\begin{array}{l}\text { Ball-on-disk } \\
\text { High } \\
\text { Temperature } \\
\text { Tribometer }\end{array}$ & $5 \mathrm{~N}$ & $5 \mathrm{~cm} / \mathrm{s}$ & $\begin{array}{l}25 \text { and } \\
400{ }^{\circ} \mathrm{C}\end{array}$ & $\begin{array}{l}\mathrm{ZnO} \mathrm{NPs} \\
\text { nificantly } \\
\text { of nanolan } \\
25{ }^{\circ} \mathrm{C} \text { in } \\
\text { they positiv } \\
\text { wear resist } \\
\text { lamellar } \mathrm{W}\end{array}$ & $\begin{array}{l}\text { did not sig- } \\
\text { hange } \mathrm{COF} \\
\text { ellar } \mathrm{WS}_{2} \text { at } \\
\text { ir, whereas } \\
\text { ely impact on } \\
\text { nce of nano- } \\
\mathrm{S}_{2} \text { at } 400{ }^{\circ} \mathrm{C}\end{array}$ & [141] \\
\hline $\mathrm{MoS}_{2}$ and $\mathrm{SiO}_{2}$ & $\begin{array}{l}\text { The layer } \\
\text { thickness of } \\
\mathrm{MoS}_{2} \text { is } \\
90 \mathrm{~nm}^{\text {The }} \\
\mathrm{SiO}_{2} \mathrm{NPs} \\
\text { have an aver- } \\
\text { age particle } \\
\text { diameter of } \\
30 \mathrm{~nm} .\end{array}$ & $\begin{array}{c}0.2,0.5 \\
0.7 \text { and } \\
1.0 \mathrm{wt} \%\end{array}$ & $\begin{array}{c}\text { EOT5\# } \\
\text { engine oil }\end{array}$ & $\begin{array}{l}\text { Ball-on-flat } \\
\text { tribometer }\end{array}$ & $\begin{array}{c}1,3,5, \\
\text { and } 8 \mathrm{~N}\end{array}$ & $0.03 \mathrm{~m} / \mathrm{s}$ & & $\begin{array}{r}\text { Exhibit } \\
\text { lubricatio }\end{array}$ & $\begin{array}{l}\text { excellent } \\
\text { properties }\end{array}$ & [39] \\
\hline $\begin{array}{l}\mathrm{Cu} / \mathrm{MoS}_{2} \\
\mathrm{Ag} / \mathrm{MoS}_{2}\end{array}$ & & & $\begin{array}{l}\text { LITOL and } \\
\text { VNIINP }\end{array}$ & $\begin{array}{c}\text { Ball-on-disk } \\
\text { High } \\
\text { Temperature } \\
\text { Tribometer }\end{array}$ & $5 \mathrm{~N}$ & $5 \mathrm{~cm} / \mathrm{s}$ & & $\begin{array}{r}\text { Changed } \\
\text { initial g } \\
\text { essentially } \\
\text { wear } r\end{array}$ & $\begin{array}{l}\text { COF of the } \\
\text { rease and } \\
\text { improved its } \\
\text { sistance }\end{array}$ & [144] \\
\hline $\begin{array}{c}\mathrm{Al}_{2} \mathrm{O}_{3} / \mathrm{TiO}_{2} \\
\text { nanocomposites }\end{array}$ & $75 \mathrm{~nm}$ & $\begin{array}{c}0,0.05 \\
0.1,0.5 \\
\text { and } 1 \mathrm{wt}^{\%} \%\end{array}$ & $\begin{array}{c}\text { pristine } \\
\text { lubricating oil }\end{array}$ & $\begin{array}{l}\text { Friction-abras } \\
\text { ion testing } \\
\text { machine }\end{array}$ & $\begin{array}{l}147 \text { and } \\
200 \mathrm{~N}\end{array}$ & $\begin{array}{l}1,200 \text { and } \\
1,450 \mathrm{rpm}\end{array}$ & $75^{\circ} \mathrm{C}$ & $\begin{array}{r}\text { Exhibit s } \\
\text { better tr } \\
\text { perform } \\
\text { lubric }\end{array}$ & $\begin{array}{l}\text { gnificantly } \\
\text { bological } \\
\text { nce of the } \\
\text { ting oil }\end{array}$ & $\begin{array}{r}{[148,} \\
149]\end{array}$ \\
\hline $\begin{array}{l}\text { Nano- } \mathrm{Cu} / \\
\text { Graphene oxide } \\
\text { nanocomposite }\end{array}$ & $5-10 \mathrm{~nm}$ & $0.05 \mathrm{wt} \%$ & paraffin oil & $\begin{array}{l}\text { Four-ball } \\
\text { tribometer }\end{array}$ & $200 \mathrm{~N}$ & $1,200 \mathrm{rpm}$ & $\mathrm{rt}$ & $\begin{array}{l}\text { Greatly re } \\
\text { and wear }\end{array}$ & $\begin{array}{l}\text { luce friction } \\
\text { as lubricant }\end{array}$ & {$[150]$} \\
\hline
\end{tabular}


(Continued)

\begin{tabular}{|c|c|c|c|c|c|c|c|c|c|c|}
\hline \multicolumn{4}{|c|}{ Nanolubricant details } & \multirow[b]{2}{*}{ Tribometer } & \multicolumn{3}{|c|}{ Conditions } & \multicolumn{2}{|c|}{ Tribological test results } & \multirow[b]{2}{*}{ Ref. } \\
\hline Nanomaterials & Grain size & $\begin{array}{c}\text { Concentra } \\
\text { tion }\end{array}$ & Base oil & & Load & Speed & $\begin{array}{c}\text { Tem- } \\
\text { perature }\end{array}$ & $\begin{array}{c}\text { Wear } \\
\text { reduction, } \\
\%\end{array}$ & $\begin{array}{c}\text { Friction } \\
\text { reduction, } \\
\%\end{array}$ & \\
\hline $\begin{array}{c}\mathrm{TiO}_{2}, \mathrm{CuO}, \mathrm{Al}_{2} \mathrm{O}_{3}, \\
\text { MWNTs }\end{array}$ & $\begin{array}{l}<21 \mathrm{~nm} \\
<50 \mathrm{~nm} \\
<50 \mathrm{~nm} \\
6-9 \mathrm{~nm} \\
\text { by } 5 \mu \mathrm{m}\end{array}$ & $\begin{array}{c}0.01 \text { to } \\
0.10 \mathrm{wt} \%\end{array}$ & $\begin{array}{c}\text { greases Mobilgrease } \\
28 \text { (PAO syntethic } \\
\text { fluid) and Uniflor } \\
8623 \mathrm{~B} \\
\text { (Perfluoropolyether) }\end{array}$ & $\begin{array}{l}\text { Four-ball } \\
\text { tribotester }\end{array}$ & $40 \mathrm{kgf}$ & $600 \mathrm{rpm}$ & $75^{\circ} \mathrm{C}$ & $\begin{array}{r}\text { Up to } 20^{\circ} \\
\text { using } \mathrm{TiO}_{2} \text {. } \\
\text { up to a } 14 \\
\text { Both } \mathrm{M} \\
\mathrm{Al}_{2} \mathrm{O}_{3} \text { inc }\end{array}$ & $\begin{array}{l}\text { o reduction } \\
\text { CuO showed } \\
\text { o reduction. } \\
\text { WNTs and } \\
\text { eased wear }\end{array}$ & {$[151]$} \\
\hline Oleic acid/La- $\mathrm{TiO}_{2}$ & $20 \mathrm{~nm}$ & $0.25 \mathrm{wt} \%$ & rapeseed oil & $\begin{array}{l}\text { Four-ball } \\
\text { friction and } \\
\text { wear tester }\end{array}$ & $392 \mathrm{~N}$ & $1,200 \mathrm{rpm}$ & & $\begin{array}{r}\text { NPs coul } \\
\text { improve a } \\
\text { friction } \\
\text { capacities o }\end{array}$ & $\begin{array}{l}\text { markedly } \\
\text { ti-wear and } \\
\text { reducing } \\
\text { rapeseed oil. }\end{array}$ & {$[152]$} \\
\hline
\end{tabular}

mechanisms primarily include the formation of a tribo-film or an adsorption film. In particular, the tribological properties of the rare-earth compounds $\mathrm{CeVO}_{4}, \mathrm{Y}_{2} \mathrm{O}_{3}, \mathrm{La}(\mathrm{OH})_{3}$, and $\mathrm{LaF}_{3}$ NPs used as FMs in lubricants have been widely studied [153-155]. Cerium oxide $(\approx 90 \mathrm{~nm})$ should also be noted, which was blended in paraffin oil and used as a nanolubricant [156]. Rare-earth compounds can obviously prolong oil life, enhance machine antiwear capacity by $2-4$ times, and improve the load-carrying capacity of lubricating grease by $10 \%-100 \%$. Moreover, the synergistic lubrication effect of rare-earth compounds and other additives is more pronounced [157].

Nanolubricants based on rare-earth compounds are listed in Table 5.

\section{Factors influencing the tribological properties of nanolubricants}

The size, morphology, surface functionalization, and concentration of NPs are among the most influential factors on the tribological properties of nanolubricants.

\subsection{Effect of nanoparticle size}

The tribological characteristics of nanolubricants directly depend on NP size. In particular, it determines their internal mechanical and physico-chemical properties,

Table 5 Rare-earth compounds as lubricant additives.

\begin{tabular}{|c|c|c|c|c|c|c|c|c|c|c|}
\hline \multicolumn{4}{|c|}{ Nanolubricant details } & \multirow[b]{2}{*}{ Tribometer } & \multicolumn{3}{|c|}{ Conditions } & \multicolumn{2}{|c|}{ Tribological test results } & \multirow{2}{*}{ Ref. } \\
\hline Nanomaterials & Grain size & Concentration & Base oil & & Load & Speed & $\begin{array}{c}\text { Tem- } \\
\text { perature }\end{array}$ & $\begin{array}{c}\text { Wear } \\
\text { reduction, \% }\end{array}$ & $\begin{array}{l}\text { Friction } \\
\text { reduction, \% }\end{array}$ & \\
\hline $\mathrm{CeO}_{2}$ & $10 \mathrm{~nm}$ & Up to $50 \mathrm{wt} \%$ & $\begin{array}{l}\text { Mixed oil of } \\
350 \mathrm{SN} \text { and } \\
650 \mathrm{SN}(1: 1, \\
\text { weight ratio) }\end{array}$ & & & $\begin{array}{c}1,450 \\
\mathrm{rpm}\end{array}$ & $25^{\circ} \mathrm{C}$ & \multicolumn{2}{|c|}{$\begin{array}{l}\text { Tribological properties were } \\
\text { significantly improved. }\end{array}$} & {$[158]$} \\
\hline $\begin{array}{c}\text { Hydroxides NPs } \\
\text { (Mg/Al/Ce } \\
\text { LDHs) }\end{array}$ & $190.1 \mathrm{~nm}$ & $\begin{array}{l}0.5 \mathrm{~g} \mathrm{LDHs} \text { per } \\
100 \mathrm{ml} \text { oil }\end{array}$ & $\begin{array}{l}\text { Diesel engine oil } \\
\text { (CD 15W-40) }\end{array}$ & $\begin{array}{c}\text { MS-10JR } \\
\text { four-ball } \\
\text { friction tester }\end{array}$ & $392 \mathrm{~N}$ & $\begin{array}{l}1,200 \\
\mathrm{rpm}\end{array}$ & $\mathrm{Rt}$ & & $\begin{array}{c}\text { Decreased to } \\
0.083(27.2 \%)\end{array}$ & {$[153]$} \\
\hline \multirow[t]{2}{*}{$\mathrm{LaF}_{3}$} & $10-30 \mathrm{~nm}$ & $0.08 \mathrm{wt} \%$ & $\begin{array}{l}\text { Fluoro } \\
\text { silicone oil }\end{array}$ & $\begin{array}{l}\text { Four-ball } \\
\text { machine }\end{array}$ & $300 \mathrm{~N}$ & $\begin{array}{c}1,450 \\
\mathrm{rpm}\end{array}$ & $25^{\circ} \mathrm{C}$ & \multicolumn{2}{|c|}{$\begin{array}{l}\text { When the load is } 500,600 \text { and } \\
700 \mathrm{~N} \text {, the wear scar diameter is } \\
\text { reduced by } 17 \%, 43 \% \text { and } 42 \% \text {, } \\
\text { respectively }\end{array}$} & [159] \\
\hline & $10-30 \mathrm{~nm}$ & $0.40 \mathrm{wt} \%$ & Liquid paraffin & $\begin{array}{c}\text { MS-10JR } \\
\text { four-ball } \\
\text { friction tester }\end{array}$ & $300 \mathrm{~N}$ & $\begin{array}{c}1,450 \\
\mathrm{rpm}\end{array}$ & $25^{\circ} \mathrm{C}$ & $\begin{array}{r}\text { Excellent ant } \\
\text { friction-reduc } \\
\text { rises }\end{array}$ & $\begin{array}{l}\text {-wear and good } \\
\text { ng ability. COF } \\
0.108 \text {. }\end{array}$ & [154] \\
\hline $\begin{array}{l}\text { Stearic acid- } \\
\text { capped } / \mathrm{CeBO}_{3}\end{array}$ & $8 \mathrm{~nm}$ & $2.0 \mathrm{wt} \%$ & Rapeseed oil & $\begin{array}{l}\text { Four-ball } \\
\text { tribo-tester }\end{array}$ & $392 \mathrm{~N}$ & $\begin{array}{c}1,500 \\
\mathrm{rpm}\end{array}$ & $\mathrm{Rt}$ & $\begin{array}{r}\text { Outstandin } \\
\text { friction-reduct } \\
\text { capacity o }\end{array}$ & $\begin{array}{l}\text { in enhancing } \\
\text { on and anti-wear } \\
\text { rapeseed oil }\end{array}$ & {$[160]$} \\
\hline
\end{tabular}


which, in turn, affect their tribological properties. For materials in the size range of $100 \mathrm{~nm}$ or higher, hardness increases as particle size decreases owing to an increase in the number of dislocation pileups for crystals (Hall-Petch regime). In this regime, hardness increases linearly with the inverse square root of particle size. At critical grain sizes, usually below $10 \mathrm{~nm}$, nanomaterials become softer as size decreases (inverse Hall-Petch regime). If NP hardness exceeds the hardness of the tribo-pair material, the result is indentation and scratching. For example, the high hardness (8-9 Mohs) of nano- $\mathrm{Al}_{2} \mathrm{O}_{3}$ compared to the metal substrate leads to abrasive wear and re-agglomeration of the NPs [125]. Therefore, in the design of a nanolubricant, it is necessary to consider the relationship between the size and hardness of NPs.

In the choice of a suitable NP size, an important parameter is the ratio of the root-mean-square roughness of the lubricant surface to the NP radius. That is, NP-based lubrication systems must remain in the contact zone during loading and shearing to protect friction surfaces. If their size is overly large compared to the characteristic roughness length scale of the shearing surfaces, the NPs will not deposit on the contact zone, which will lead to poor lubrication. However, when the characteristic roughness length scale is significantly larger than the NP radius, the valleys between asperities of the shearing surfaces can be filled with NPs.

Finally, the homogeneity of the nanolubricant composition, which largely controls its tribological characteristics, depends heavily on colloidal stability. Dispersion stability is a function of NP size, which is the main requirement for the correct composition of the nanolubricant. An important parameter for determining dispersion stability is sedimentation rate, which can be calculated using the Stokes law:

$$
v_{z}=\frac{2\left(\rho_{\mathrm{NP}}-\rho_{\mathrm{F}}\right) g r^{2}}{9 \mu}
$$

where $v_{z}$ is the settling velocity, $\rho_{\mathrm{NP}}$ is NP density, $\rho_{\mathrm{F}}$ is the density of the fluid, $g$ is gravity, $r$ is NP radius, and $\mu$ is the viscosity of the fluid.

According to the Stokes law, smaller size implies better dispersion stability and tribological behavior.

In Ref. [161], a nanolubricant based on CuO NPs was added to synthetic oil at three concentration and size levels: $0.1,0.25$, and $0.5 \mathrm{wt} \%$, and $2.5,4.4$, and $8.7 \mathrm{~nm}$, respectively [161]. It was demonstrated that a low NP concentration reduces wear and contributes to a smooth surface, whereas a large plastic deformation is observed at a high concentration. Furthermore, the smallest NP size corresponded to the smallest COF. In general, the best results were obtained for a nanolubricant with a concentration of $0.1 \mathrm{wt} \%$ and an NP size of $2.5 \mathrm{~nm}$.

\subsection{Effect of nanoparticle morphology}

The shape of NPs used as lubricant additives is another important parameter for nanolubricant design, because it directly determines the pressure experienced by the NPs during loading. There are five types of NP shapes: granular, onion, sheet, spherical, and tube. According to the statistics (Fig. 2), most NP shapes are spherical, followed by granular, sheet, onion, and nanotube.

After nucleation, crystalline particle structures tend to evolve so that surface energy may be minimized, which leads to a spherical shape. Onion morphology is described as a spherical shape on the outside and a lamellar structure inside. If the onion morphology is stable, it is closer to spherical morphology. Otherwise, it will exfoliate and become a sheet-like morphology. The advantages of onion structure lie in the absence of dangling bonds and spherical shape.

Onion, leaf, and spherical morphologies exhibit excellent tribological characteristics. Spherically shaped NPs exhibit high load capacity and EP characteristics owing to their ball bearing effect, which can change friction characteristics from sliding to rolling, thus reducing friction $[148,149]$. The spherical shape of

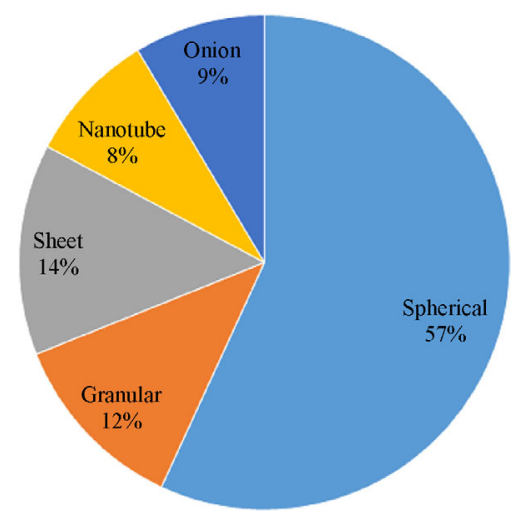

Fig. 2 Statistics of NP morphology [15]. 
NPs leads to point contact with the counter surface. Line contact is associated with nanosheets, whereas planar contact is a feature of nanoplatelets.

It is of interest to investigate the tribological properties of single-crystalline $\alpha$ - and $\beta-\mathrm{MnO}_{2}$ nanorods as nanoadditives in green lubricants [162]. The minimum friction torque was observed for $\alpha-\mathrm{MnO}_{2}$-added palm oil, followed by $\beta-\mathrm{MnO}_{2}$-added palm oil, and pure palm oil (Fig. 3(a)). Even though $\beta-\mathrm{MnO}_{2}$ nanorods exhibited a decrease in COF by approximately $15 \%$, $\alpha-\mathrm{MnO}_{2}$ nanorods are even better nanoadditives, with a reduction of up to $30 \%$ (Fig. 3(b)). Such an increase in anti-wear capacity arises primarily from the interplay between the rolling action and the formation of a protective layer by the corresponding quasi-1D $\mathrm{MnO}_{2}$ polymorphs.

Another example illustrating the significance of a nanostructure is the layered structure of NPs of transition metal dichalcogenides, which is more suitable for reducing friction by forming a tribo-film [163, 164]. Figure 4 shows the molecular structure of $\mathrm{MoS}_{2}$ as an example of a layered crystal structure.

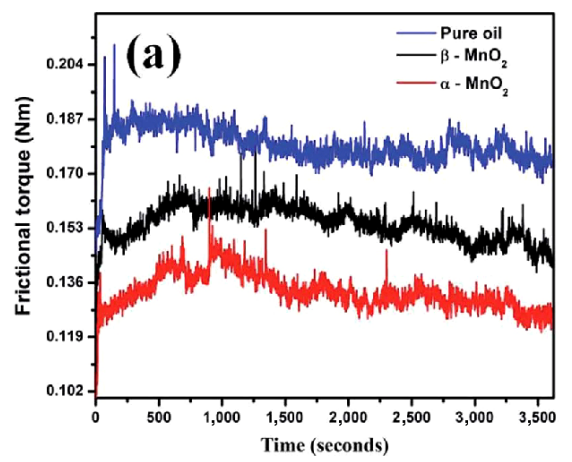

Compared to typical transition metal dichalcogenides, IFs have been developed that are layered compounds with a hollow polyhedral structure [165]. They exhibit excellent tribological behavior under severe contact conditions and tend to form tribo-films on friction surfaces [127]. Such solid IF-NPs can use additional "exfoliation" lubrication mechanisms. In addition to their layered structure, sulfur plays an important role in the interaction between particles and lubricant molecules.

Although materials such as $\mathrm{MoS}_{2}$ have been studied for some time, various other 2D nanomaterials have appeared as an alternative to friction modification $[165,166]$.

\subsection{Effect of surface functionalization}

The functionalization of the NP surface is used to regulate the colloidal stability of the NP dispersion and to increase the lubricity of most layers of NPs. It is well known that non-functionalized NPs tend to aggregate in inert non-polar liquids such as hydrocarbon. Aggregation is usually prevented by protecting

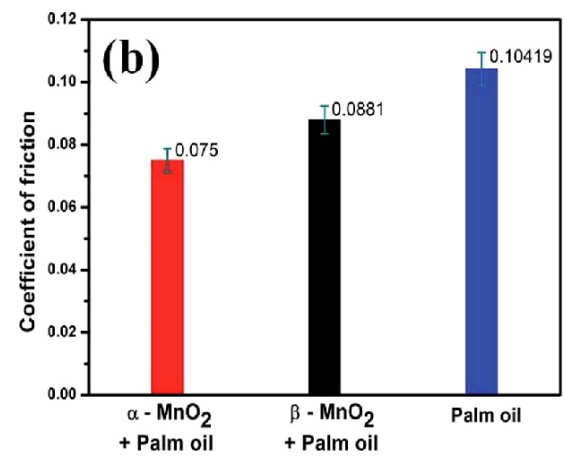

Fig. 3 (a) Frictional torque with respect to time observed using four-ball test technique, (b) $\mathrm{COF}$ of pure palm oil, $\beta$ - $\mathrm{MnO}_{2}$-added palm oil, and $\alpha-\mathrm{MnO}_{2}$-added palm oil.

(a)

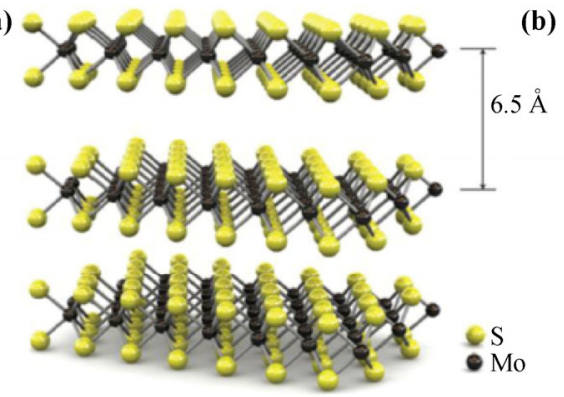

(b)

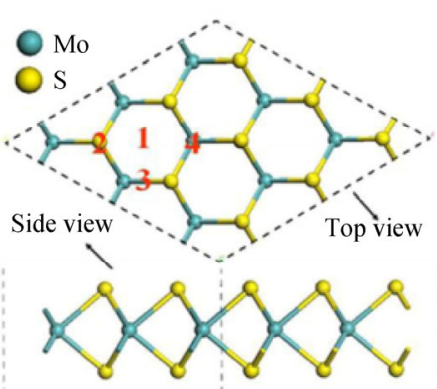

Fig. 4 (a) Three-dimensional representation of the structure of $\mathrm{MoS}_{2}$. Single layers, $6.5 \AA$ thick, can be extracted using scotch tape-based micromechanical cleavage. (b) Optimized structures of $\mathrm{MoS}_{2}$ monolayer with four adsorption sites: (1) hollow site, (2) top site of the S atom, (3) Mo-S bridge site, and (4) top site of the Mo atom [165]. 
NPs by steric or electrostatic stabilization, which typically involves coating the NPs with a polymer or surfactant. As a rule, the functionalization of the NP surface is necessary to increase the colloidal stability and homogeneous distribution of NPs in the base oil.

It should be noted that functionalized NPs have better lubricating properties compared to bare NPs because the latter experience material transfer when they come into direct contact with shearing surfaces and prevent cold-welding of the shearing surfaces. In addition, functionalized NPs prevent the transfer of material between them and cold-welding between the shearing surfaces. Of great importance is the fact that functionalized NPs have a hybrid structure with a rigid inner core and a soft outer shell. This synergistic combination provides NPs with a rigid internal shape and a slippery fluid-like surface (Fig. 5, left). Ultimately, such NPs allow higher load carrying capacity, without reducing lubricity (Fig. 5, right) [167].

Finely dispersed $\mathrm{Cu}$ NPs covered by surfactants were used as an additive to fully-formulated engine oils [81]. The tribological process of formation of protective films on the metal surface includes the accumulation of polar molecules by absorption to produce an FM film and mechanochemical processes comprising a combination of redox reactions and a third body formation. The oil-soluble Cu NPs obtained by surface modification with tetradecyl hydroxamic acid have been used as environmentally friendly oil additives that could remarkably improve anti-wear and friction reduction performance. Cu NPs can deposit and fill up micropits and grooves on steel friction surfaces under a higher load, and consequently they significantly reduce steel pair wear by self-repairing worn surfaces [80]. One should note triangular copper nanoplates prepared with cetyltrimethylammonium bromide as the capping agent [82]. As an additive for lubricants, nanoplates are responsible for the formation of a film deposit at the interface of a friction pair and a $12 \%$ drop in COF of the lubricant and an $82.2 \%$ drop in wear loss. Cu NPs surface-capped by dioctylamine dithiocarbamate [83] or alkanethiols [84] were used as an additive in liquid paraffin. They have excellent anti-wear and friction-reduction properties owing to the deposition of $\mathrm{Cu}$ NPs, with a low melting point on the worn steel surface, which results in the formation of a self-repairing protective layer on it.

$\mathrm{Ni}$-based nanolubricants with oleylamine and oleic acid as surface-capping agents in poly-alpha-olefin as a base oil [87] or a synergistic lubricant system with a solid liquid [168] exhibit good anti-wear behavior even at low $\mathrm{Ni}$ concentrations $(0.05 \mathrm{wt} \%)$. This is because surface-capped Ni NPs in nanolubricants can release highly active $\mathrm{Ni}$ nanocores as well as $\mathrm{O}-$ and $\mathrm{N}$-containing organic modifying agents, which can easily form a boundary lubricating film on sliding steel surfaces.

Two types of thiolated ligands, namely, 4-(tertbutyl)benzylthiol and dodecanthiol, were used to modify oil-suspended Ag NPs in the ranges 1-3 nm and 3-6 nm [77]. The organic surface layer successfully suspended Ag NPs in PAO base oil with concentrations up to $0.19-0.50 \mathrm{wt} \%$, depending on the particle type. Using Ag NPs in the base oil reduces friction by up to $35 \%$ and wear by up to $85 \%$ in boundary lubrication. NPs modified with a ligand of the first type resulted in lower COF than NPs modified with a second-type ligand, whereas larger NPs (3-6 nm) had better wear
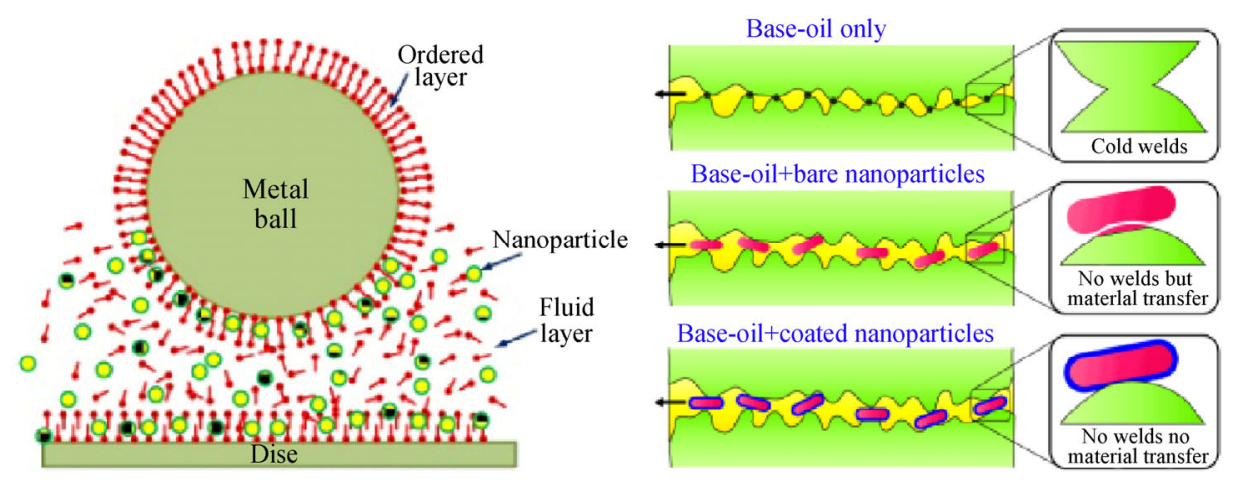

Fig. 5 (left) Core-shell structure of NP on the disc; (right) comparison of the effect of three types of lubrication conditions on cold-welding and material transfer behavior of a tribo-pair. 
protection than smaller NPs (1-3 nm). It is important that the molecular structure of the organic ligand can exert a dominant influence on friction behavior, whereas NP size may be more influential in wear protection. Wear protection in boundary lubrication is due to the formation of a $50-100 \mathrm{~nm}$ thick silver-rich tribo-film on the worn surface.

It is of interest to study the tribological properties of $\mathrm{CuO}$ NPs dual-coated with sodium oleate and alkylphenol polyoxyethylene ether [163, 164]. The COF and wear scar diameter of deionized water in the presence of dual-coated CuO NPs are significantly reduced, and excellent tribological properties under a certain load are obtained at the optimum concentration of the dual-coated $\mathrm{CuO}$ NPs. $\mathrm{CuO}$ nanorods stabilized with ionic liquids exhibit excellent friction reduction (15\%-43\%) and improved anti-wear properties $(26 \%-$ 43\%) compared to PEG 200 and 10W-40 engine oil [169]. The increase in the lubricity of $\mathrm{CuO}$ nanorods is due to their good dispersion stability and rolling mechanism. One should note the use of tiny CuO NPs with low concentrations as EP additives in synthetic oil [56]. NPs with an average size of $5 \mathrm{~nm}$ were coated with oleic acid and added to poly-alpha-olefin oil using a toluene dispersant. It was demonstrated that it is possible to reduce COF and wear using tiny NPs, as well as to reduce the percentage of the $\mathrm{CuO}$ addition in the lubricating oil. The wear and friction properties of a suspension of $\mathrm{CuO}(50 \mathrm{~nm})$ NPs modified with oleic acid in liquid paraffin were studied [170]. After modification, the lowest COF (0.123) was obtained at $3 \% \mathrm{CuO}$ and the highest value $(0.158)$ at $0.2 \% \mathrm{CuO}$. Nanolubricants based on castor and paraffin oil with CuO NPs, modified with a surfactant sodium dodecyl sulfate, as an additive in the regime of boundary lubrication were studied in Ref. [171]. The maximum wear reduction was $28.3 \%$ and $22.2 \%$, whereas COF was reduced by $34.6 \%$ and $17.3 \%$ at optimum NP concentration in the former and latter oils, respectively. A significant improvement in the weld load was observed for both nanolubricants.

Oleic acid surface-modified ZnO NPs dispersed in 60SN base oil [100], poly-alpha olefin, or diisooctyl sebacate [127] significantly reduced friction and wear. Interestingly, when the amount of oleic acid added was $8 \mathrm{wt} \%$ and $\mathrm{ZnO}$ NPs was $0.5 \mathrm{wt} \%$, COF and the average diameter of the wear scars were minimal, and the nanolubricant exhibited the best friction-reducing and anti-wear properties. It is of interest to use nanolubricants based on multiwalled carbon nanotubes and $\mathrm{ZnO}$ NPs with a volume fraction of $0.005 \%$ and $0.02 \%$, respectively, dispersed along with Gum Arabic surfactant in SAE 20W40 engine oil [172].

It should be noted that $\mathrm{TiO}_{2}$ NPs modified by tetra(2-ethylhexyl)-thiuramdisulfide and di(2ethylhexyl)-thiophonedisulfide can be completely welldispersed in the base oil, with no significantly negative effect on anti-friction properties [121, 122]. It is important that functionalized $\mathrm{TiO}_{2}$ NPs exhibit better anti-wear and friction-reducing properties in base oil compared to non-coated $\mathrm{TiO}_{2}$ NPs. Aqueous suspensions containing various concentrations of $\mathrm{TiO}_{2} \mathrm{NPs}(50 \mathrm{~nm})$, in which sodium polyacrylate is used as the dispersant, have good anti-wear and friction reduction properties as well as load-carrying capacity [173].

Oleic acid was used as a surfactant to improve the stability of oil-based $\mathrm{SnO}_{2}$ nanofluid, reducing COF by up to $65.4 \%$ and the wear volume loss by up to $43.7 \%$ [123]. A tribosintered or embedded patchy film containing tin was observed inside the wear track, which protected the surface from wear and lowered COF. In addition, $\mathrm{SnO}_{2} \mathrm{NPs}$ can roll or slide between two friction surfaces to prevent adhesive wear. $\mathrm{CeO}_{2}$ NPs $(\approx 90 \mathrm{~nm})$ were used as additives in castor oil with four different concentrations in the range $0.1 \%$ $1.0 \% w / v$, with sodium dodecyl sulfate as a dispersant [174]. The maximum reduction in the wear scar diameter was $37.4 \%$ at the optimum concentration of $\mathrm{CeO}_{2}$.

Solid sphere-like $\mathrm{MoS}_{2}$ NPs improve the tribological properties of dioctyl sebacate (DOS) more than commercial micro- $\mathrm{MoS}_{2}$ [133]. It is important that $\mathrm{MoS}_{2} \mathrm{NPs}$ bind to DOS molecules, promoting the solidification of DOS on the surface of $\mathrm{MoS}_{2}$ NPs and the formation of fiber-like solids aggregated into a net-like structure (Fig. 6). A large number of DOS molecules are captured in the net-like structure, forming an adsorption film that reduces friction and wear.

\subsection{Effect of nanoparticle concentration}

Concentration is another important factor that affects the lubrication characteristics of nanolubricants [35]. Typically, the addition of NPs is effective in reducing friction and wear, even at concentrations below $1 \mathrm{wt} \%$ [59] and above $2 \mathrm{wt} \%$ [170], indicating that NPs do 


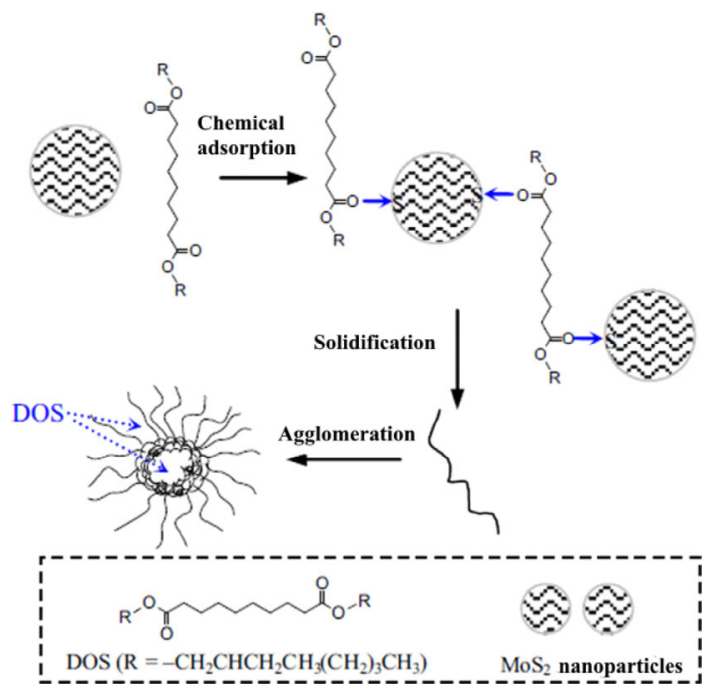

Fig. 6 Formation of adsorption layer using $\mathrm{MoS}_{2}$ NPs [133].

not have an ideal concentration. In addition, there is no predictable relationship between the concentration and the effect of the nanoadditive on friction and wear. It should be noted that there is an optimum concentration at which COF is minimal. However, it is highly dependent on the system because the lubricant composition must be adjusted for each operating condition [175]. For example, the same $\mathrm{MoS}_{2} \mathrm{NPs}$ show different suitable concentrations for two different base lubricants, i.e., $0.58 \mathrm{wt} \%$ for mineral oil and $0.53 \mathrm{wt} \%$ for coconut oil [35]. Furthermore, a fixed optimal concentration of $0.5 \mathrm{wt} \%$ of $\mathrm{CuO}$ NPs and $\mathrm{ZnO}$ NPs was established for mineral, synthetic, and vegetable oils [126]. A study of the tribo-performance of cerium oxide $(\approx 90 \mathrm{~nm})$ NPs in paraffin oil with a concentration change from 0.1 to $1.0 \% w / v$ demonstrated that a concentration of $0.25 \% w / v$ is optimal in antiwear and anti-friction tests [171]. At this concentration, the maximum reduction in the wear scar diameter was $26.1 \%$ and the average COF was reduced by $29.6 \%$. For nanolubricants based on sunflower oil and two types of the nanoadditives, that is, $\mathrm{CuO}$ and $\mathrm{CeO}_{2}$, with different concentrations from $0.10 \%$ to $0.50 \% w / v$, a concentration of $0.10 \% w / v$ for the nanoadditives is optimal owing to least wear scar and COF [176]. A higher NP concentration degrades the base oil performance. Hafnium doped into diamond-like thin films exhibited low COF and excellent wear resistance at the optimum $0.42 \%$ Hf concentration [177].

It was suggested $[178,179]$ that NPs reduce the real area of contact, and consequently reduce the friction in boundary lubrication. That is, the particles in contact will keep the surfaces apart around the particles, leading to a decrease in the real contact area (Fig. 7) and thus to a decrease in COF.

When more concentrated nanolubricants are used, more particles will come into contact, which explains the monotonic decrease in COF compared to the particle concentration in the tests.

The obtained results demonstrate that each type of NP should be analyzed considering both the previously mentioned factors (size, morphology, surface functionalization, and concentration) and the application conditions (temperature, load, and slip speed), as well as the nature of the base lubricant.

\section{Lubrication mechanisms}

The investigation of lubrication mechanisms is considered a decisive parameter for a complete understanding of nanolubricant tribology. However, the definition of active mechanisms remains a subject of discussion in several studies on metal-containing nanoadditives to lubricating oils. A number of mechanisms have been proposed using surface analysis techniques to explain the increase in lubrication. These mechanisms include ball bearing, the formation of a protective film, mending, and polishing.

To study the lubrication mechanisms for lubricating oils enriched with NPs, a number of methods for characterizing surfaces have been used [59, 124, 126, $127,180,181]$. However, it was noted that owing to the existence of different lubrication mechanisms by nanolubricants, these surface analysis tools are not sufficient to distinguish the role of NPs among

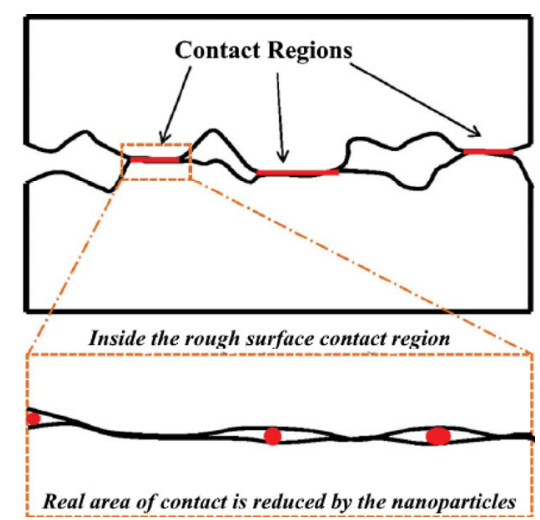

Fig. 7 Mechanism of reduction of the real area of contact by NPs. 
these mechanisms. The combinations of lubrication mechanisms include the direct action of NPs (ball bearing/tribo-film formation) and secondary surface enhancement (mending/polishing). For example, the examination of the performance of a four-stroke diesel engine with the addition of $\mathrm{Al}_{2} \mathrm{O}_{3} \mathrm{NPs}$ to the engine oil (SAE15W40) demonstrated signs of ball bearing and surface polishing, which are responsible for the improvement of the tribological properties of the oil [182].

\subsection{Ball bearing effect}

Spherical and quasi-spherical NPs generally function as tiny ball bearings that roll into the contact zone and change sliding friction to a mixture of sliding and rolling friction (Fig. 8).

In particular, the rolling friction of sphere-like $\mathrm{CuO}$ NPs at the contact surface could improve the tribological properties of the base lubricant [126, 184]. Introducing a nanolubricant may result in superior product quality owing to the rolling action of NPs between sliding surfaces, thus preventing surface contact [185]. Analysis of $\mathrm{ZnO}$ composite submicrospheres with $\mathrm{Al}_{2} \mathrm{O}_{3} \mathrm{NPs}$ as additives for lubricating oils has demonstrated that rolling friction becomes dominant instead of sliding friction, and these composite particles squeezed into grooves on the friction surfaces can reduce wear [186]. Copper oxide NPs convert sliding friction into rolling friction, thereby reducing the effective COF [128].

\subsection{Protective film formation}

The protective film on the tested surfaces is also called a tribo-film. Tribo-films and near-surface materials

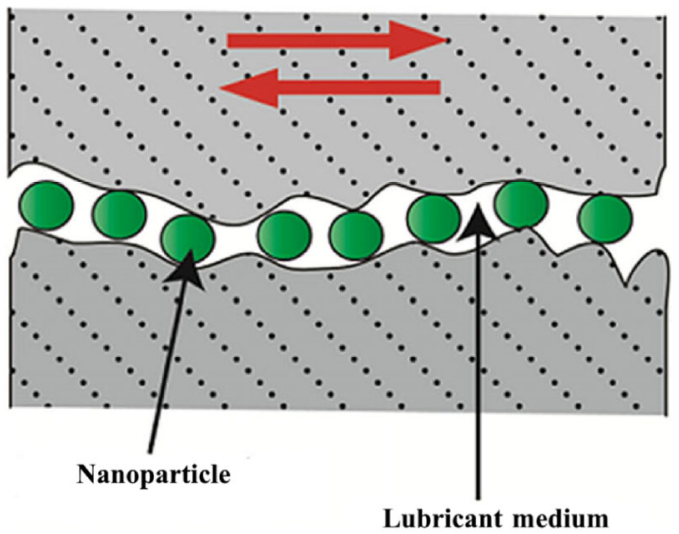

Fig. 8 Ball bearing mechanism by NP-based lubrication [183]. determine the tribological behavior of friction surfaces. Film formation is initiated by a reaction between the treated material and additives under environmental conditions or tribo-sintering $[13,187]$. There are several experimental studies that describe the mechanism of tribo-film formation for excellent lubrication. The rate of tribo-film formation should be higher than the wear removal rate to protect worn surfaces [188-190]. Self-replenishment is necessary for maintaining a tribo-film with sufficient adhesion to the substrate and internal cohesion to withstand the friction during boundary lubrication. NPs play a vital role in the formation of tribo-films on contact interfaces to improve engine performance and combustion through various mechanisms [191]. Tribological characteristics are associated with the mechanical strength and thickness of the tribo-film produced on worn surfaces [192]. As presented in some studies [56, 59, 119, 170], the efficiency of metallic NPs is attributed to their deposition on worn surfaces forming a thin layer, generally softer than the substrate, capable of reducing friction through smaller sliding resistance and of protecting the substrate from wear by preventing metal-to-metal contact.

Figure 9 shows the patterns of tribo-film formation, which not only provides surface protection but also protects the material from crack propagation by reducing the friction between the asperities [13].

An investigation of the influence of $\mathrm{Cu}$ NPs on the tribological properties of attapulgite base grease demonstrated that under lubrication, a smoother and more compact tribo-film was formed on the friction surface $[72,73]$. It primarily consisted of $\mathrm{Cu}, \mathrm{FeO}, \mathrm{Fe}_{2} \mathrm{O}_{3}$, $\mathrm{FeOOH}, \mathrm{CuO}$, and $\mathrm{SiO}_{2}$, and the content of iron oxides and silicate oxide formed in tribo-film increases by the introduction of $\mathrm{Cu}$ NPs.

In addition, wear debris $\left(\mathrm{Fe}_{3} \mathrm{O}_{4}\right.$ particles) deposited in the structure of tribo-film was observed in other studies [193]. The use of Cu-doped muscovite composite particles as lubricant additives leads to the formation of tribo-film primarily consisting of $\mathrm{O}, \mathrm{Si}, \mathrm{Fe}$, $\mathrm{Cu}$, as well as $\mathrm{Al}$ elements on the block worn surface, thereby further reducing friction and wear [194].

It should be noted that a smoother and more compact tribo-film is formed on the worn surface, which is responsible for further friction and wear reduction, by using nanolubricants based on vegetable 


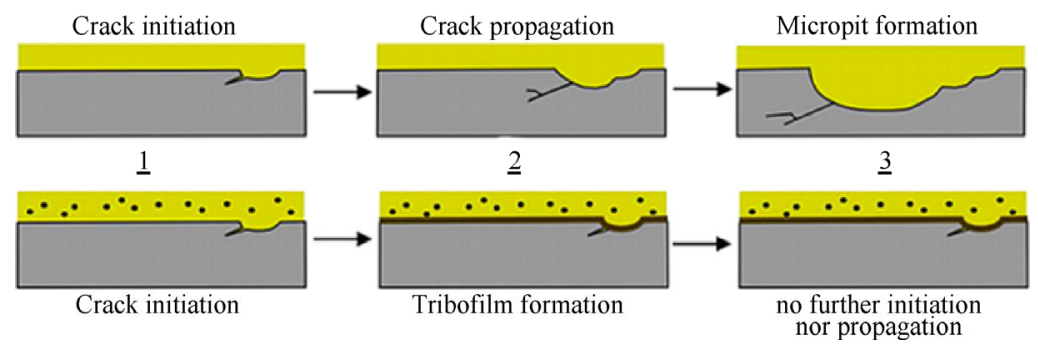

Fig. 9 Lubrication by base oil alone (top), and tribo-film formation and surface protection by NPs (bottom) [13].

lubricants with the addition of $\mathrm{ZnO}$ and $\mathrm{CuO}$ NPs [101].

It is of interest to study the formation of tribo-films using molecular dynamics simulation [195-197]. It was demonstrated that owing to the adsorption layer around NPs, nanolubricant molecules become more organized and compact compared to base oil. In addition, soft $\mathrm{Cu}$ NPs are deformed by the structural elements of the nanolubricant film, which provides good support for the lubricating film.

\subsection{Mending effect}

The mending or self-repairing effect is characterized by NPs deposition on friction surfaces and mass loss compensation. During this phenomenon, NPs deposit on the wear surface and fill the scars and grooves of the friction surface to reduce abrasion.

The study of the effects of the nanolubricants made of $\mathrm{CuO}$ and $\mathrm{Al}_{2} \mathrm{O}_{3}$ NPs on the surface quality of the forging process demonstrated that nanolubricants significantly improve surface roughness compared to conventional lubricants [198]. Suspensions of surfacemodified $\mathrm{CuO}$ NPs in bio-based lubricant exhibited high EP characteristics in terms of load wear index and low cylinder liner wear owing to the surface mending effect of NPs [185].

\subsection{Polishing effect}

The polishing effect, also called smoothing effect, is manifested when the roughness of the lubricating surface is reduced by abrasive treatment with NPs. In tribological contacts, NPs can fill the gaps of rough asperities that can act as reservoirs of solid lubricants (NPs) in contact. This process of filling up rough valleys is called smoothing out process. This "artificial smoothing" or polishing mechanism results in improved tribological characteristics owing primarily to reduced surface roughness [183].

\subsection{Friction mechanism of IF-nanoparticles}

The lubrication mechanisms of IF-NPs as FMs include the following: rolling, sliding, and third body effects (Fig. 10) [183].

Rolling friction implies that NPs will roll between two sliding surfaces, which requires a spherical shape and a stable structure. In the case of sliding, the IF-NPs serve as a spacer and eliminate the metal/metal contact between the asperities of both surfaces under slightly higher loads. For the case of third body effect, the exfoliation of the IF-NPs and their external layers are gradually transferred to the roughness of the friction surfaces, providing easy shearing under high loads, where the third body can be considered a mixture of oil, NPs, and wear particles.

Poorly crystallized particles have better lubricating properties owing to their tendency to exfoliate, forming a tribo-film consisting of sheet-like particles on the surface. In particular, such sheet-like particles include $\mathrm{MoS}_{2}$ nanoplatelets and $\mathrm{Y}_{2} \mathrm{O}_{3}$ [199]. To understand the lubrication mechanisms of sheet-like NPs, there are two types of interactions that play a decisive role in determining the frictional behavior [167]. For $\mathrm{MoS}_{2}$ owing to its weak interlayer van der Walls forces, two
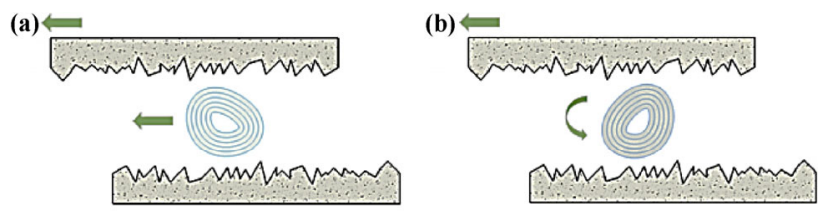

(c)

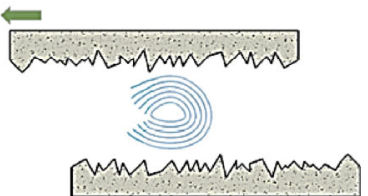

Fig. 10 Three main friction mechanisms of multilayered IF NP: (a) sliding, (b) rolling, (c) exfoliation [15]. 
adjacent layers are easily exfoliated under a shear force, and sliding movement of the adjacent layer leads to friction reduction. For other 2D NPs with relatively strong interlayer van der Walls forces, in particular $\mathrm{Y}_{2} \mathrm{O}_{3}$ [200], it is difficult for layers to exfoliate. Another interaction associated with the outer layer and the substrate is determined by the surface energy of the basal plane and the property of the environment. It should be emphasized that the number of layers and interlayer spacing affect tribological performance. When the number of layers decreases, other problems appear, for example, the puckering and wrinkle effects, inclination angles [199], and interlayer spacing [201].

\section{Concluding remarks}

Analysis of existing data on the use of metal-containing nanomaterials as lubricant additives indicates significant progress on several problems regarding nanolubricants; it also demonstrates that this is an active research field. It can be confidently concluded that the development of this interesting field of nanomaterials science has reached its peak in the accumulation of experimental facts and their theoretical interpretation and generalization, although this is only the tip of the iceberg in terms of the potential for their application. As objects of research, several new types of metalcontaining nanomaterials were presented. The main emphasis in these studies is on the use of environmentally friendly technologies, the possibility of mass production, and efficiency, which will make these materials promising for future industrial applications. However, it is unfortunately impossible to determine the correlations between composition, size, morphology, surface functionalization, NP concentration, and nanolubricant properties, which in several respects impedes the development of a scientifically ground approach to structuring these nanomaterials and predicting their promising properties. To date, although a large number of experimental studies have been carried out on nanoparticle as additives for lubricating oils, several aspects of their tribological behavior have not yet been fully understood. Furthermore, it should be noted that new groups of researchers are involved in this field of nanomaterials science.

The following are important tasks in the deve- lopment of the field, the accomplishment of which would give an opportunity to discover the general principles of nanolubricants:

- Maintaining their long-term dispersion stability.

To stabilize NPs in various lubricating base oils, several combinations of surfactants/NPs should be investigated, as well as surface functionalization methods.

- Investigating their compatibility with lubricant additives, such as detergents, dispersants, antioxidants, viscosity improvers, and corrosion inhibitors.

- The relationship between their molecular structure and their tribological characteristics should be further discussed and applied as a guide for the molecular design of new nanolubricants.

- Regarding environmental protection, it is necessary to develop environmentally friendly nanolubricants that do not contain sulfur and phosphorus, without reducing wear and friction characteristics.

- The development of multifunctional lubricant additives with excellent anti-wear, friction-reduction, extreme pressure, and antioxidant properties will be the main trend in this field, and their joint mechanisms of action should be investigated.

- The tribological mechanism of nanolubricants should be studied and examined in more detail using modern analytical methods as well as molecular simulation.

Open Access: This article is licensed under a Creative Commons Attribution 4.0 International License, which permits use, sharing, adaptation, distribution and reproduction in any medium or format, as long as you give appropriate credit to the original author(s) and the source, provide a link to the Creative Commons licence, and indicate if changes were made.

The images or other third party material in this article are included in the article's Creative Commons licence, unless indicated otherwise in a credit line to the material. If material is not included in the article's Creative Commons licence and your intended use is not permitted by statutory regulation or exceeds the permitted use, you will need to obtain permission directly from the copyright holder. To view a copy of this licence, visit http://creativecommons.org/licenses/ by/4.0/. 


\section{References}

[1] Zhang Y, Chromik R. Self-Lubricating Composites. Menezes P L, Rohatgi P K, Omrani E, eds. Springer, Cham, 2018.

[2] Tribocatalysis, Tribochemistry, and Tribocorrosion. Kajdas C, Hiratsuka K, eds. CRC, Pan Stanford, 2018.

[3] Gnanasekaran D, Chavidi VP. Vegetable Oil based Biolubricants and Transformer Fluids. Springer, Singapore, 2018.

[4] Darminesh S P, Sidik N A C, Najafi G, Mamat R, Ken T L, Asako Y. Recent development on biodegradable nanolubricant: A review. Int J Heat Mass Transf 86: 159-165 (2017)

[5] Tang Z, Li S. A review of recent developments of friction modifiers for liquid lubricants (2007-present). Curr Opin Solid State Mater Sci 18: 119-139 (2014)

[6] Xiao H, Liu S. 2D nanomaterials as lubricant additive: A review. Mater Des 135: 319-332 (2017)

[7] Liu L, Zhou M, Li X, Jin L, Su G, Mo Y, Li L, Zhu H, Tian Y. Research progress in application of 2D materials in liquid-phase lubrication system. Materials 11: 1314 (2018)

[8] Shahnazar S, Bagheri S, Hamid S B A. Enhancing lubricant properties by nanoparticle additives. Int J Hydrogen Energy 41: 3153-3170 (2016)

[9] Minami I. Molecular science of lubricant additives. Appl Sci 7: 445 (2017)

[10] Mathew J, Joy J, George S C. Potential applications of nanotechnology in transportation: A review. J King Saud Univ Sci, https://doi.org/10.1016/j.jksus.2018.03.015 (2018)

[11] Kotia A, Rajkhowa P, Rao G S, Ghosh S K. Thermophysical and tribological properties of nanolubricants: A review. Heat Mass Transfer 54: 3493-3508 (2018)

[12] Guo W, Yin J, Qiu H, Guo Y, Wu H, Xue M. Friction of low-dimensional nanomaterial systems. Friction 2: 209-225 (2014)

[13] Gulzar M, Masjuki H H, Kalam M A, Varman M, Zulkifli N W M, Mufti R A, Zahid R. Tribological performance of nanoparticles as lubricating oil additives. J Nanopart Res 18: 223 (2016)

[14] Dang R K, Goyal D, Dhami S S, Chauhan A. Effect of nanoparticles based lubricants on static thermal behaviour of journal bearings: A review. Res J Eng Tech 8: 149-153 (2017)

[15] Dai W, Kheireddin B, Gao H, Liang H. Roles of nanoparticles in oil lubrication. Tribol Int 102: 88-98 (2016)

[16] Ali M K A, Xianjun H. Improving the tribological behavior of internal combustion engines via the addition of nanoparticles to engine oils. Nanotechnol Rev 4: 347-358 (2015)

[17] Kaviyarasu T, Vasanthan B. Improvement of tribological and thermal properties of engine lubricant by using nano- materials. J Chem Pharm Sci (JCHPS) 7: 208-211 (2015)

[18] Khond V W, Kriplani V. Effect of nanofluid additives on performances and emissions of emulsified diesel and biodiesel fueled stationary $\mathrm{Ci}$ engine: A comprehensive review. Renew Sustain Energy Rev 59: 1338-1348 (2016)

[19] Kong L, Sun J, Bao Y. Preparation, characterization and tribological mechanism of nanofluids. RSC $A d v$ 7: 12599 (2017)

[20] Peña-Parás L, Maldonado-Cortés D, Taha-Tijerina J. Handbook of Ecomaterials. Martínez L, Kharissova O, Kharisov B, eds. Springer, Cham, 2019

[21] Zhang S-W. Green tribology: Fundamentals and future development. Friction 1: 186-194 (2013)

[22] Zhang S-W. Recent developments of green tribology. Surf Topogr: Metrol Prop 4: 023004 (2016)

[23] Zhai W, Srikanth N, Kong L B, Zhou K. Carbon nanomaterials in tribology. Carbon 119: 150-171 (2017)

[24] Shahmohamadi S H, Rahmani R, Rahnejat H, Garner C P, Balodimos N. Thermohydrodynamics of lubricant flow with carbon nanoparticles in tribological contacts. Tribol Int 113: 50-57 (2017)

[25] Wang B, Tang W, Liu X, Huang Z. Synthesis of ionic liquid decorated muti-walled carbon nanotubes as the favorable water-based lubricant additives. Appl Phys A: Mater Sci Proces 123: 680 (2017)

[26] He A, Huang S, Yun J-H, Jiang Z, Stokes J R, Jiao S, Wang L, Huang H. Tribological Characteristics of aqueous graphene oxide, graphitic carbon nitride, and their mixed suspensions. Tribol Lett 66: 42 (2018)

[27] Gusain R, Mungse H P, Kumar N, Ravindran T R, Pandian R, Sugimura H, Khatri O P. Covalently attached graphene-ionic liquid hybrid nanomaterials: synthesis, characterization and tribological application. J Mater Chem A 4: 926-937 (2016)

[28] Liu Y, Mateti S, Li C, Liu X, Glushenkov A M, Liu D, Li L H, Fabijanic D, Chen Y. Synthesis of composite nanosheets of graphene and boron nitride and their lubrication application in oil. Adv Eng Mater 20: 1700488 (2018)

[29] Sheng Y, Yang J, Wang F, Liu L, Liu H, Yan C, Guo Z. Sol-gel synthesized hexagonal boron nitride/titania nanocomposites with enhanced photocatalytic activity. Appl Surf Sci 465: 154-163 (2019)

[30] Zhang R, Zhao J, Pu J, Lu Z. First-principles investigation on the tribological properties of h-BN bilayer under variable load. Tribol Lett 66: 124 (2018)

[31] Liu X, Xu N, Li W, Zhang M, Chen L, Lou W, Wang X. Exploring the effect of nanoparticle size on the tribological properties of $\mathrm{SiO}_{2} /$ polyalkylene glycol nanofluid under different lubrication conditions. Tribol Int 109: 467-472 (2017) 
[32] Singh S K, Chattopadhyaya S, Pramanik A, Kumar S, Gupta N. Influence of nano-particle on the wear behaviour of thin film coatings. A review. Int J Appl Eng Res 13: 4053-4058 (2018)

[33] Patil S J, Patil D P, Shrotri A P, Patil V P. A review on effect of addition of nano particles on tribological properties of lubricants. Int J Mech Eng Technol (IJMET) 5: 120-129 (2014)

[34] Uflyand I E, Zhinzhilo V A, Lapshina L S, Novikova A A, Burlakova V E, Dzhardimalieva G I. Conjugated thermolysis of metal chelate monomers based on cobalt acrylate complexes with polypyridyl ligands and tribological performance of nanomaterials obtained. ChemistrySelect 3: 8998-9007 (2018)

[35] Koshy C P, Rajendrakumar P K, Thottackkad M V. Evaluation of the tribological and thermo-physical properties of coconut oil added with $\mathrm{MoS}_{2}$ nanoparticles at elevated temperatures. Wear 330-331: 288-308 (2015)

[36] Zin V, Agresti F, Barison S, Colla L, Fabrizio M. Influence of $\mathrm{Cu}, \mathrm{TiO}_{2}$ nanoparticles and carbon nano-horns on tribological properties of engine oil. J Nanosci Nanotechnol 15: 3590-3598 (2015)

[37] Abdullah M I H C, Abdollah M F B, Tamaldin N, Amiruddin H, Mat Nuri N R, Gachot C, Kaleli H. Effect of hexagonal boron nitride nanoparticles as an additive on the extreme pressure properties of engine oil. Ind Lubr Tribol 68: 441-445 (2016)

[38] Çelik O N, Ay N, Göncü Y. Effect of nano hexagonal boron nitride lubricant additives on the friction and wear properties of AISI 4140 steel. Part Sci Technol 31: 501-506 (2013)

[39] Xie H, Jiang B, He J, Xia X, Pan F. Lubrication performance of $\mathrm{MoS}_{2}$ and $\mathrm{SiO}_{2}$ nanoparticles as lubricant additives in magnesium alloy-steel contacts. Tribol Int 93: 63-70 (2016)

[40] Uflyand I E, Dzhardimalieva G I, Nanomaterials Preparation by Thermolysis of Metal Chelates. Springer, Cham, 2018

[41] Ealias A M, Saravanakumar M P. A review on the classification, characterisation, synthesis of nanoparticles and their application. IOP Conf Ser: Mater Sci Eng 263: 032019 (2017)

[42] Rao B G, Mukherjee D, Reddy B M. Nanostructures for Novel Therapy. Synthesis, Characterization and Applications. Ficai D, Grumezescu A, eds. Elsevier, Amsterdam, 2017

[43] Qiao S-Z, Liu J, Max Lu G Q. Modern Inorganic Synthetic Chemistry, 2nd edn. Xu R, Xu Y, eds. Elsevier, Amsterdam, 2017

[44] Nanomaterials and Nanocomposites: Zero- to ThreeDimensional Materials and Their Composites. Visakh P M, Morlanes M J M, eds. Wiley, Weinheim, 2016

[45] Amosov A P. Nanomaterials of SHS technology for tribological applications: A review. Russ J Non-Ferrous Metals 58: 530-539 (2017)

[46] Ali M K A, Abdelkareem M A A, Elagouz A, Essa F A, Xianjun H. mini review on the significance nano-lubricants in boundary lubrication regime. Int $J$ Biosensors \& Bioelectronics 2: 42-43 (2017)

[47] Ali M K A, Xianjun H, Mai L, Qingping C, Turkson R F, Bicheng C. Improving the tribological characteristics of piston ring assembly in automotive engines using $\mathrm{Al}_{2} \mathrm{O}_{3}$ and $\mathrm{TiO}_{2}$ nanomaterials as nano-lubricant additives. Tribol Int 103: 540-554 (2016)

[48] Alahmer A. Influence of using emulsified diesel fuel on the performance and pollutants emitted from diesel engine. Energy Convers Manag 73: 361-369 (2013)

[49] Ali M K A, Xianjun H, Turkson R F, Ezzat M. An analytical study of tribological parameters between piston ring and cylinder liner in internal combustion engines. Proc Inst Mech Eng Part K: J Multi-Body Dyn 230: 329-349 (2016)

[50] Guo D, Xie G, Luo J. Mechanical properties of nanoparticles: Basics and applications. J Phys D: Appl Phys 47: 013001 (2013)

[51] Ali M K A, Xianjun H, Elagouz A, Essa F A, Abdelkareem $\mathrm{M}$ A A. Minimizing of the boundary friction coefficient in automotive engines using $\mathrm{Al}_{2} \mathrm{O}_{3}$ and $\mathrm{TiO}_{2}$ nanoparticles. $J$ Nanopart Res 18: 377 (2016)

[52] Zareh-Desari B, Davoodi B. Assessing the lubrication performance of vegetable oil-based nano-lubricants for environmentally conscious metal forming processes. J Clean Prod 135: 1198-1209 (2016)

[53] Gulzar M, Masjuki H H, Varman M, Kalam M A, Mufti R A, Zulkifli N W M, Yunus R, Zahid R. Improving the AW/EP ability of chemically modified palm oil by adding $\mathrm{CuO}$ and $\mathrm{MoS}_{2}$ nanoparticles. Tribol Int 88: 271-279 (2015)

[54] Alves S M, Mello V S, Faria E A, Camargo A P P. Nanolubricants developed from tiny $\mathrm{CuO}$ nanoparticles. Tribol Int 100: 263 (2016)

[55] Sanukrishna S S, Vishnu S, Krishnakumar T S, Prakash M J. Effect of oxide nanoparticles on the thermal, rheological and tribological behaviours of refrigerant compressor oil: An experimental investigation. Int J Refrig 90: 32-45 (2018)

[56] Ingole S, Charanpahari A, Kakade A, Umare S S, Bhatt D $\mathrm{V}$, Menghani J. Tribological behavior of nano $\mathrm{TiO}_{2}$ as an additive in base oil. Wear 301: 776-785 (2013)

[57] Asnida M, Hisham S, Awang N W, Amirruddin A K, Noor M M, Kadirgama K, Ramasamy D, Najafi G, Tarlochan F. Copper (II) oxide nanoparticles as additive in engine oil to increase the durability of piston-liner contact. Fuel 212: 656-667 (2018) 
[58] Luo T, Wei X, Huang X, Huang L, Yang F. Tribological properties of $\mathrm{Al}_{2} \mathrm{O}_{3}$ nanoparticles as lubricating oil additives. Ceram Int 40: 7143-7149 (2014)

[59] Padgurskas J, Rukuiza R, Prosycevas I, Kreuvaitis R. Tribological properties of lubricant additives of $\mathrm{Fe}, \mathrm{Cu}$ and Co nanoparticles. Tribol Int 60: 224-232 (2013)

[60] Borda F L G, de Oliveira S J R, Lazaro L M S M, Leiróz A $\mathrm{J}$ K. Experimental investigation of the tribological behavior of lubricants with additive containing copper nanoparticles. Tribol Int 117: 52-58 (2018)

[61] Garg P, Kumar A, Thakre G D, Arya P K, Jain A K. Investigating efficacy of $\mathrm{Cu}$ nano-particles as additive for bio-lubricants. Macromol Symp 376: 1700010 (2017)

[62] Gaur M K, Singh S K, Sood A, Chauhan D S. Advances in Design, Simulation and Manufacturing. Ivanov V, Rong Y, Trojanowska J, Venus J, Liaposhchenko O, Zajac J, Pavlenko I, Edl M, Perakovic D, eds. Springer, Cham, 2019

[63] Ghaednia H, Babaei H, Jackson R L, Bozack M J, Khodadadi J. The effect of nanoparticles on thin film elastohydrodynamic lubrication. Appl Phys Lett 103: 263111 (2013)

[64] Kalyani R, Chockalingam G, Gurunathan K. Tribological aspects of metal and metal oxide nanoparticles. Adv Sci Eng Medicine 8: 228-232 (2016)

[65] Meng H N, Zhang Z Z, Zhao F X, Qiu T, Zhu X, Lu X J. Tribological behaviours of $\mathrm{Cu}$ nanoparticles recovered from electroplating effluent as lubricant additive. Tribol-Mater Surf Interfaces 9: 46-53 (2015)

[66] Li Y, Liu T T, Zhang Y, Zhang P, Zhang S. Study on the tribological behaviors of copper nanoparticles in three kinds of commercially available lubricants. Ind Lubr Tribol 70: 519-526 (2018)

[67] Najan A B, Navthar R R, Gitay M J. Experimental Investigation of tribological properties using nanoparticles as modifiers in lubricating oil. Int Res J Eng Technol (IRJET) 4: 1125-1129 (2017)

[68] Songmei Y, Xuebo H, Guangyuan Z, Amin M. A novel approach of applying copper nanoparticles in minimum quantity lubrication for milling of Ti-6Al-4V. Adv Prod Eng Manag 12: 139-150 (2017)

[69] Zhang X-M, Yang X-P, Ouyang P. Research progress in copper-containing micro and nano particles as lubricating additives. Xiandai Huagong/Modern Chem Ind 34: 53-56 (2014)

[70] Yang G, Zhang Z, Zhang S, Yu L, Zhang P. Synthesis and characterization of highly stable dispersions of copper nanoparticles by a novel one-pot method. Mater Res Bull 48: 1716-1719 (2013)

[71] $\mathrm{Hu} \mathrm{H}$, Peng H, Ding G. Nucleate pool boiling heat transfer characteristics of refrigerant/nanolubricant mixture with surfactant. Int J Refrig 36: 1045 (2013)

[72] Zhang C, Zhang S, Song S, Yang G, Yu L, Wu Z, Li X, Zhang P. Preparation and tribological properties of surfacecapped copper nanoparticle as a water-based lubricant additive. Tribol Lett 54: 25-33 (2014)

[73] Nan F, Xu Y, Xu B, Gao F, Wu Y, Li Z. Effect of Cu Nanoparticles on the tribological performance of attapulgite base grease. Tribol Trans 58: 1031-1038 (2015)

[74] Thapliyal P, Kumar A, Thakre G D, Jain A K. Investigation of rheological parameters of lubricants and contact fatigue behavior of steel in the presence of $\mathrm{Cu}$ nano-particles. Macromol Symp 376: 1700011 (2017)

[75] Song H, Huang J, Jia X, Sheng W. Facile synthesis of core-shell Ag@C nanospheres with improved tribological properties for water-based additives. New J Chem 42: 87738782 (2018)

[76] Beckford S, Cai J, Chen J, Zou M. Use of Au NanoparticleFilled PTFE films to produce low-friction and low-wear surface coatings. Tribol Lett 56: 223-230 (2014)

[77] Kumara C, Luo H, Leonard D N, Meyer H M, Qu J. Organic-modified silver nanoparticles as lubricant additives. ACS App Mater Interface 9: 37227-37237 (2017)

[78] Zhang S, Li Y, Hu L, Feng D, Wang H. Antiwear effect of Mo and $\mathrm{W}$ nanoparticles as additives for multialkylated cyclopentanes oil in vacuum. J Tribol 139: 021607 (2016)

[79] Li Y, Zhang S, Ding Q, Li H, Qin B, Hu L. Understanding the synergistic lubrication effect of 2-mercaptobenzothiazolate based ionic liquids and Mo nanoparticles as hybrid additives. Tribol Int 125: 39-45 (2018)

[80] Flores-Castañeda M, Camps E, Camacho-López M, Muhl S, García E, Figueroa M. Bismuth nanoparticles synthesized by laser ablation in lubricant oils for tribological tests. J Alloys Compd 643: S67-S70 (2015)

[81] Gonzalez-Rodriguez P, Van Den Nieuwenhuijzen K J H, Lette W, Schipper D J, Ten Elshof J E. Tribochemistry of bismuth and bismuth salts for solid lubrication. ACS Appl Mater Interfaces 8: 7601-7606 (2016)

[82] Scherge M, Böttcher R, Kürten D, Linsler D. Multi-Phase friction and wear reduction by copper nanopartices. Lubricants 4: 36 (2016)

[83] Wang X L, Yin Y L, Zhang G N, Wang W Y, Zhao K K. Study on antiwear and repairing performances about mass of nano-copper lubricating additives to 45 Steel. Phys Procedia 50: 466-472 (2013)

[84] Wang J, Guo X, He Y, Jiang M, Sun R. The synthesis and tribological characteristics of triangular copper nanoplates as a grease additive. $R S C A d v$ 7: 40249-40254 (2017)

[85] Yang G, Zhang Z, Zhang S, Yu L, Zhang P, Hou Y. Preparation and characterization of copper nanoparticles surface-capped by alkanethiols. Surf Interface Anal 45: 1695-1701 (2013) 
[86] Zhang Y, Xu Y, Yang Y, Zhang S, Zhang P, Zhang Z. Synthesis and tribological properties of oil-soluble copper nanoparticles as environmentally friendly lubricating oil additives. Ind Lubr Tribol 67: 227-232 (2015)

[87] Zhang Y, Zhang S, Zhang P, Yang G, Zhang Z. Advances in Tribology. Darji P H, ed. InTech, 2016.

[88] Chen Y, Zhang Y, Zhang S, Yu L, Zhang P, Zhang Z. Preparation of nickel-based nanolubricants via a facile in situ one-step route and investigation of their tribological properties. Tribol Lett 51: 73-83 (2013)

[89] Abad M D, Sánchez-López J C. Tribological properties of surface-modified Pd nanoparticles for electrical contacts. Wear 297: 943-951 (2013)

[90] Zhang S, Hu L, Feng D, Wang H. Anti-wear and frictionreduction mechanism of $\mathrm{Sn}$ and $\mathrm{Fe}$ nanoparticles as additives of multialkylated cyclopentanes under vacuum condition. Vacuum 87: 75-80 (2013)

[91] Kedzierski M A. Effect of concentration on $\mathrm{R} 134 \mathrm{a} / \mathrm{Al}_{2} \mathrm{O}_{3}$ nanolubricant mixture boiling on a reentrant cavity surface. Int $J$ Refrig 49: 36-48 (2015)

[92] Shaari M Z, Roselina N R N, Kasolang S, Hyie K M, Murad M C, Bakar M A A. Investigation of tribological properties of palm oil biolubricant modified nanoparticles. Jurnal Teknologi 76: 69-73 (2015)

[93] Arumugam S, Sriram G. Preliminary study of nano- and microscale $\mathrm{TiO}_{2}$ additives on tribological behavior of chemically modified rapeseed oil. Tribol Trans 56: 797-805 (2013)

[94] Fangsuwannarak K, Triratanasirichai K. Improvements of palm biodiesel properties by using nano- $\mathrm{TiO}_{2}$ additive, exhaust emission and engine performance. Rom Rev Precis Mech Opt Mechatron 43: 111-118 (2013)

[95] Laad M, Jatti V K S. Titanium oxide nanoparticles as additives in engine oil. J King Saud Univ - Eng Sci 30: 116-122 (2018)

[96] Xia W, Zhao J, Wu H, Zhao X, Zhang X, Xu J, Jiao S, Jiang Z. Effects of oil-in-water based nanolubricant containing $\mathrm{TiO}_{2}$ nanoparticles in hot rolling of 304 stainless steel. Procedia Eng 207: 1385-1390 (2017)

[97] Trajano M F, Moura E I F, Ribeiro K S B, Alves S M. Study of oxide nanoparticles as additives for vegetable lubricants. Mater Res 17: 1124-1128 (2014)

[98] Bhaumik S, Maggirwar R, Datta S, Pathak S D. Analyses of anti-wear and extreme pressure properties of castor oil with zinc oxide nano friction modifiers. Appl Surf Sci 449: 277-286 (2018)

[99] Essa F A, Zhang Q, Huang X, Ibrahim A M M, Ali M K A, Abdelkareem M A A, Elagouz A. Improved friction and wear of M50 steel composites incorporated with $\mathrm{ZnO}$ as a solid lubricant with different concentrations under different load. J Mater Eng Perform 26: 4855-4866 (2017)
[100] Suresh Kumar V P, Manikandan N, Subakaran C, Sterbin Jeso Y G. An experimental effect of $\mathrm{ZnO}$ nanoparticles in SAE 20W50 oil. Int Res J Eng Technol (IRJET) 5: 1069-1073 (2018)

[101] Ran X, Yu X, Zou Q. Effect of particle concentration on tribological properties of $\mathrm{ZnO}$ nanofluids. Tribol Trans 60: $154-158$ (2017)

[102] Thottackkad M V, Rajendrakumar P K, Prabhakaran Nair $\mathrm{K}$. Experimental studies on the tribological behaviour of engine oil (SAE15W40) with the addition of $\mathrm{CuO}$ nanoparticles. Ind Lubr Tribol 66: 289-297 (2014)

[103] Arumugam S, Sriram G. Synthesis and characterization of rapeseed oil bio-lubricant dispersed with nano copper oxide: its effect on wear and frictional behavior of piston ringcylinder liner combination. Proc Inst Mech Eng Part J 228: 1308 (2014)

[104] Yang P, Zhao X, Liu Y, Lai X. Preparation and tribological properties of dual-coated $\mathrm{CuO}$ nanoparticles as water based lubricant additives. J Nanosci Nanotechnol 16: 9683 (2016)

[105] Trivedi K, Parekh K, Upadhyay R V. Nanolubricant: Magnetic nanoparticle based. Mater Res Express 4: 114003 (2017)

[106] Zhou G, Zhu Y, Wang X, Xia M, Zhang Y, Ding H. Sliding tribological properties of $0.45 \%$ carbon steel lubricated with $\mathrm{Fe}_{3} \mathrm{O}_{4}$ magnetic nanoparticle additives in base oil. Wear 301: 753-757 (2013)

[107] Pisal A S, Chavan D S. Experimental investigation of tribological properties of engine oil with $\mathrm{CuO}$ nanoparticles. Int J Theor Appl Res Mech Eng (IJTARME) 3: 34-38 (2014)

[108] Kashyap A, Harsha A P. Tribological studies on chemically modified rapeseed oil with $\mathrm{CuO}$ and $\mathrm{CeO}_{2}$ nanoparticles. Proc Inst Mech Eng Part J: J Eng Tribol 230: 1562-1571 (2016)

[109] Liu H, Zhang Y, Zhang S, Chen Y, Zhang P, Zhang Z. Preparation and evaluation of tribological properties of oil-soluble rice-like $\mathrm{CuO}$ nanoparticles. Ind Lubr Tribol 67: 276-283 (2015)

[110] Manjunatha G, Anil Kumar T. Influence of heat treatment temperatures on wear and hardness properties of copper oxide nanoparticle reinforced composites. Int Res J Eng Technol (IRJET) 3: 251-254 (2016)

[111] Dai W, Lee K, Sinyukov A M, Liang H. Effects of vanadium oxide nanoparticles on friction and wear reduction. $J$ Tribol 139: 061607 (2017)

[112] Thottackkad M V, Rajendrakumar P K, Prabhakaran N K. Tribological analysis of surfactant modified nanolubricants containing $\mathrm{CeO}_{2}$ nanoparticles. Tribol-Mater Surf Interfaces 8: $125-130$ (2014)

[113] Zulhanafi P, Syahrullail S, Faridzuan M M. Tribological performance of palm kernel oil added with nanoparticle 
copper oxide using fourball tribotester. Jurnal Teknologi 79: 53-59 (2017)

[114] Xia W, Zhao J, Wu H, Jiao S, Zhao X, Zhang X, Xu J, Jiang Z. Analysis of oil-in-water based nanolubricants with varying mass fractions of oil and $\mathrm{TiO}_{2}$ nanoparticles. Wear 396-397: 162-171 (2018)

[115] Xia W, Zhao J, Cheng X, Sun J, Wu H, Yan Y, Jiao S, Jiang Z. Study on growth behaviour of oxide scale and its effects on tribological property of nano- $\mathrm{TiO}_{2}$ additive oil-inwater lubricant. Wear 376-377: 792-802 (2017)

[116] Wu H, Zhao J, Cheng X, Xia W, He A, Yun J H, Huang S, Wang L, Huang H, Jiao S, Jiang Z. Friction and wear characteristics of $\mathrm{TiO}_{2}$ nano-additive water-based lubricant on Ferritic stainless steel. Tribol Int 117: 24-38 (2018)

[117] Gu Y, Zhao X, Liu Y, Lv Y. Preparation and tribological properties of dual-coated $\mathrm{TiO}_{2}$ nanoparticles as water-based lubricant additives. J Nanomater 2014: Article ID 785680 (2014)

[118] Binu K G, Shenoy B S, Rao D S, Pai R. A variable viscosity approach for the evaluation of load carrying capacity of oil lubricated journal bearing with $\mathrm{TiO}_{2}$ nanoparticles as lubricant additives. Procedia Mater Sci 6: 1051-1067 (2014)

[119] Zulkifli N W M, Kalam M A, Masjuki H H, Yunus R. Experimental analysis of tribological properties of biolubricant with nanoparticle additive. Procedia Eng 68: 152-157 (2013)

[120] Laad M, Ponnamma D, Sadasivuni K K. Tribological studies of nanomodified mineral based multi-grade engine oil. Int J Appl Eng Res 12: 2855-2861 (2017)

[121] Ilie F, Covaliu C. Tribological properties of the lubricant containing titanium dioxide nanoparticles as an additive. Lubricants 4: 12 (2016)

[122] Zaimovskaya T A, Oganesova E Yu, Kuzmina G N, Ezhov A A, Ivanov V K, Parenago O P. Titanium-containing compounds as efficient triboadditives to oils. J Friction Wear 34: 487-493 (2013)

[123] Tao C, Wang B, Barber G C, Schall J D, Lan H. Tribological behaviour of $\mathrm{SnO}_{2}$ nanoparticles as an oil additive on brass. Lubr Sci 30: 247-255 (2018)

[124] Thakre A A, Thakur A. Study of behaviour of aluminium oxide nanoparticles suspended in SAE20W40 oil under extreme pressure lubrication. Ind Lubr Tribol 67: 328 (2015)

[125] Peña-Parás L, Taha-Tijerina J, Garza L, Maldonado-Cortés D, Michalczewski R, Lapray C. Effect of $\mathrm{CuO}$ and $\mathrm{Al}_{2} \mathrm{O}_{3}$ nanoparticle additives on the tribological behavior of fully formulated oils. Wear 332-333: 1256-1261 (2015)

[126] Alves S M, Barros B S, Trajano M F, Ribeiro K S B, Moura E. Tribological behavior of vegetable oil-based lubricants with nanoparticles of oxides in boundary lubrication conditions. Tribol Int 65: 28-36 (2013)

[127] Wu L, Zhang Y, Yang G, Zhang S, Yu L, Zhang P.
Tribological properties of oleic acid-modified zinc oxide nanoparticles as the lubricant additive in poly-alpha olefin and diisooctyl sebacate base oils. $R S C A d v$ 6: 69836-69844 (2016)

[128] Jatti V S, Singh T P. Copper oxide nano-particles as friction-reduction and anti-wear additives in lubricating oil. J Mech Sci Technol 29: 793-798 (2015)

[129] Gao C, Wang Y, Hu D, Pan Z, Xiang L. Tribological properties of magnetite nanoparticles with various morphologies as lubricating additives. J Nanopart Res $\mathbf{1 5}$ : 1502 (2013)

[130] Yadgarov L, Petrone V, Rosentsveig R, Feldman Y, Tenne R, Senatore A. Tribological studies of rhenium doped fullerene-like $\mathrm{MoS}_{2}$ nanoparticles in boundary, mixed and elasto-hydrodynamic lubrication conditions. Wear 297: 1103-1110 (2013)

[131] Zhou L H, Wei X C, Ma Z J, Mei B. Anti-friction performance of $\mathrm{FeS}$ nanoparticle synthesized by biological method. Appl Surf Sci 407: 21-28 (2017)

[132] Rabaso P, Ville F, Dassenoy F, Diaby M, Afanasiev P, Cavoret J, Vacher B, Le Mogne T. Boundary lubrication: influence of the size and structure of inorganic fullerenelike $\mathrm{MoS}_{2}$ nanoparticles on friction and wear reduction. Wear 320: 161-178 (2014)

[133] Xu Y, Hu E, Hu K, Xu Y, Hu X. Formation of an adsorption film of $\mathrm{MoS}_{2}$ nanoparticles and dioctylsebacate on a steel surface for alleviating friction and wear. Tribol Int 92: 172-183 (2015)

[134] Kalin M, Kogovšek J, Remškar M. Nanoparticles as novel lubricating additives in a green, physically based lubrication technology for DLC coatings. Wear 303: 480-485 (2013)

[135] Wan Q, Jin Y, Sun P, Ding Y. Rheological and tribological behaviour of lubricating oils containing platelet $\mathrm{MoS}_{2}$ nanoparticles. J Nanopart Res 16: 2386 (2014)

[136] Nazir M H, Khan Z A, Saeed A, Siddaiah A, Menezes P L. Synergistic wear-corrosion analysis and modelling of nanocomposite coatings. Tribol Int 121: 30-44 (2018)

[137] Irtegov Y, An V, Machekhina K, Lemachko N. Influence of copper nanoparticles on tribological properties of nanolamellar tungsten disulfide. Key Engineering Materials 712: 133-136 (2016)

[138] Abreu C S, Matos J, Cavaleiro A, Alves E, Barradas N P, Vaz F, Torrell M, Gomes J R. Tribological characterization of $\mathrm{TiO}_{2} / \mathrm{Au}$ decorative thin films obtained by PVD magnetron sputtering technology. Wear 330-331: 419-428 (2015)

[139] Ataie S A, Zakeri A. Improving tribological properties of $(\mathrm{Zn}-\mathrm{Ni}) /$ nano $\mathrm{Al}_{2} \mathrm{O}_{3}$ composite coatings produced by ultrasonic assisted pulse plating. $J$ Alloys Compds 674: 315-322 (2016)

[140] Meng Y, Su F, Chen Y. Effective lubricant additive of nano-Ag/MWCNTs nanocomposite produced by supercritical 
$\mathrm{CO}_{2}$ synthesis. Tribol Int 118: 180-188 (2018)

[141] Meng Y, Su F, Chen Y. Supercritical fluid synthesis and tribological applications of silver nanoparticle-decorated graphene in engine oil nanofluid. Nanofluid Sci Rep 6: 1 (2016)

[142] An V, Irtegov Y, Anisimov E, Druzyanova V, Burtsev N, Khaskelberg M. Tribological properties of nanolamellar tungsten disulfide doped with zinc oxide nanoparticles. SpringerPlus 4: 673 (2015)

[143] Anisimov E, Irtegov Y, An V, Druzyanova V, Burtsev $\mathrm{N}$, Khaskelberg M B. Use of zinc oxide nanopowder as an additive in a tribotechnical composite based on refractory metal disulfide. Key Engineering Materials 685: 539-542 (2016)

[144] An V, Anisimov E, Druzyanova V, Burtsev_N, Shulepov I, Khaskelberg M. Study of tribological behavior of $\mathrm{Cu}-\mathrm{MoS}_{2}$ and $\mathrm{Ag}-\mathrm{MoS}_{2}$ nanocomposite lubricants. SpringerPlus 5: 72 (2016)

[145] An V, Irtegov Y. Tribological Properties of nanolamellar $\operatorname{mos}_{2}$ doped with copper nanoparticles. J Nanomater 2014: Article ID 731073 (2014)

[146] Gulzar M, Masjuki H, Kalam M, Varman M, Zulkifli N, Mufti R, Zahid R, Yunus R. Dispersion stability and tribological characteristics of $\mathrm{TiO}_{2} / \mathrm{SiO}_{2}$ nanocompositeenriched biobased lubricant. Tribol Trans 60: 670-680 (2017)

[147] Zhao J, He Y, Wang Y, Wang W, Yan L, Luo J. An investigation on the tribological properties of multilayer grapheme and $\mathrm{MoS}_{2}$ nanosheets as additives used in hydraulic applications. Tribol Int 97: 14-20 (2016)

[148] Luo T, Wei X, Zhao H, Cai G, Zheng X. Tribology properties of $\mathrm{Al}_{2} \mathrm{O}_{3} / \mathrm{TiO}_{2}$ nanocomposites as lubricant additives. Ceram Int 40: 10103-10109 (2014)

[149] Ali M K A, Fuming P, Younus H A, Abdelkareem M A A, Essa F A, Elagouz A, Xianjun H. Fuel economy in gasoline engines using $\mathrm{Al}_{2} \mathrm{O}_{3} / \mathrm{TiO}_{2}$ nanomaterials as nanolubricant additives. Appl Energy 211: 461-478 (2018)

[150] Meng Y, Su F, Chen Y. Synthesis of nano-Cu/grapheme oxide composites by supercritical $\mathrm{CO}_{2}$-assisted deposition as a novel material for reducing friction and wear. Chem Eng J 281: 11-19 (2015)

[151] Peña-Parás L, Taha-Tijerina J, García A, Maldonado D, Nájera A, Cantú P, Ortiz D. Thermal transport and tribological properties of nanogreases for metal-mechanic applications. Wear 332-333: 1322-1326 (2015)

[152] Gu K, Chen B, Chen Y. Preparation and tribological properties of lanthanum- doped $\mathrm{TiO}_{2}$ nanoparticles in rapeseed oil. $J$ Rare Earths 31: 589-594 (2013)

[153] Li S, Qin H, Zuo R, Bai Z. Tribological performance of $\mathrm{Mg} / \mathrm{Al} / \mathrm{Ce}$ layered double hydroxides nanoparticles and intercalated products as lubricant additives. Appl Surf Sci 353: 643-650 (2015)

[154] Li S, Qin H, Zuo R, Bai Z. Friction properties of La-doped $\mathrm{Mg} / \mathrm{Al}$ layered double hydroxide and intercalated product as lubricant additives. Tribol Int 91: 60-66 (2015)

[155] Li Z, Hou X, Yu L, Zhang Z, Zhang P. Preparation of lanthanum trifluoride nanoparticles surface-capped by tributylphosphate and evaluation of their tribological properties as lubricant additive in liquid paraffin. Appl Surf Sci 292: 971-977 (2014)

[156] Gupta R N, Harsha A P. Tribological evaluation of calciumcopper-titanate/cerium oxide-based nanolubricants in sliding contact. Lubr Sci 30: 175-187 (2018)

[157] Liu T, Hu X, Hu E, Xu Y. Modern Mechanical Engineering. Materials Forming, Machining and Tribology. Davim J, ed. Springer, Berlin, Heidelberg, 2014

[158] Shen T, Wang D, Yun J, Liu Q, Liu X, Peng Z. Tribological properties and tribochemical analysis of nano-cerium oxide and sulfurized isobutene in titanium complex grease. Tribol Int 93: 332-346 (2016)

[159] Hou X, He J, Yu L, Li Z, Zhang Z, Zhang P. Preparation and tribological properties of fluorosilane surface-modified lanthanum trifluoride nanoparticles as additive of fluoro silicone oil. Appl Surf Sci 316: 515-523 (2014)

[160] Boshui C, Kecheng G, Jianhua F, Jiang W, Jiu W, Nan Z. Tribological characteristics of monodispersed cerium borate nanospheres in biodegradable rapeseed oil lubricant. Appl Surf Sci 353: 326-332 (2015)

[161] Alves S M, Silva e Mello V, Sinatora A. Nanolubrication mechanisms: Influence of size and concentration of $\mathrm{CuO}$ nanoparticles. Mater Perform Character 7: 20170064 (2018)

[162] Kumar N, Bhaumik S, Sen A, Shukla A P, Pathak S D. One-pot synthesis and first-principles elasticity analysis of polymorphic $\mathrm{MnO}_{2}$ nanorods for tribological assessment as friction modifiers. RSC Adv 7: 34138-34148 (2017)

[163] Spikes H. Friction modifier additives. Tribol Lett 60: 5 (2015)

[164] Sgroi M F, Asti M, Gili F, Deorsola F A, Bensaid S, Fino D, Kraft G, Garcia I, Dassenoy F. Engine bench and road testing of an engine oil containing $\mathrm{MoS}_{2}$ particles as nanoadditive for friction reduction. Tribol Int 105: 317-325 (2017)

[165] He Z, Que W. Molybdenum disulfide nanomaterials: Structures, properties, synthesis and recent progress on hydrogen evolution reaction. Appl Mater Today 3: 23-56 (2016)

[166] Berman D, Erdemir A, Sumant A V. Approaches for achieving superlubricity in two-dimensional materials. ACS Nano 12: 2122-2137 (2018)

[167] Spear J C, Ewers B W, Batteas J D. 2D-nanomaterials for 
controlling friction and wear at interfaces. Nano Today 10: 301-314 (2015)

[168] Liu Y, Xin L, Zhang Y, Chen Y, Zhang S, Zhang P. The effect of Ni nanoparticles on the lubrication of a DLC-based solid-liquid synergetic system in all lubrication regimes. Tribol Lett 65: 31 (2017)

[169] Gusain R, Khatri O P. Ultrasound assisted shape regulation of $\mathrm{CuO}$ nanorods in ionic liquids and their use as energy efficient lubricant additives. J Mater Chem A 1: 5612-5619 (2013)

[170] Asrul M, Zulkifli N W M, Masjuki H H, Kalam M A. Tribological properties and lubricant mechanism of Nanoparticle in Engine Oil. Procedia Eng 68: 320-325 (2013)

[171] Gupta R N, Harsha A P. Tribological study of castor oil with surface-modified $\mathrm{CuO}$ nanoparticles in boundary lubrication. Ind Lubr Tribol 70: 700-710 (2018)

[172] Dinesh R, Prasad M J G, Kumar R R, Santharaj N J, Raaj J S A S A, Investigation of tribological and thermophysical properties of engine oil containing nano additives. Mater Today: Proceedings 3: 45-53 (2016)

[173] Kong L, Sun J, Bao Y, Meng Y. Effect of $\mathrm{TiO}_{2}$ nanoparticles on wettability and tribological performance of aqueous suspension. Wear 376-377: 786-791 (2017)

[174] Gupta R N, Harsha A P. Antiwear and extreme pressure performance of castor oil with nano-additives. Proc Inst Mech Eng Part J: J Eng Tribol 232: 1055-1067 (2017)

[175] Azman N F, Samion S, Mat Sot M N H. Investigation of tribological properties of $\mathrm{CuO} /$ palm oil nanolubricant using pin-on-disc tribotester. Green Mater 6: 30-37 (2018)

[176] Gupta R N, Harsha A P. Friction and wear of nanoadditivebased biolubricants in steel-steel sliding contacts: A comparative study. J Mater Eng Perform 27: 648-658 (2018)

[177] Qi M, Xiao J, Gong C, Jiang A, Chen Y. Evolution of the mechanical and tribological properties of DLC thin films doped with low-concentration hafnium on $316 \mathrm{~L}$ steel. $J$ Phys D: Appl Phys 51: 025301 (2018)

[178] Ghaednia H, Jackson R L, Khodadadi J M. Experimental analysis of stable $\mathrm{CuO}$ nanoparticle enhanced lubricants. $J$ Exp Nanosci 10: 1-18 (2015)

[179] Ghaednia H, Jackson R L. The effect of nanoparticles on the real area of contact, friction and wear. J Tribol 135 : 041603 (2013)

[180] Ettefaghi E, Ahmadi H, Rashidi A, Seyed S M, Mahshad A. Experimental evaluation of engine oil properties containing copper oxide nanoparticles as a nanoadditive. Int $J$ Ind Chem 4: 28 (2013)

[181] Gulzar M, Masjuki H H, Kalam M A, Varman M, Zulkifli N W M. Antiwear behavior of $\mathrm{CuO}$ nanoparticles as additive in bio-based lubricant. Key Engineering Materials 748: 166-170 (2017)
[182] Kotia A, Borkakoti S, Ghosh S K. Wear and performance analysis of a 4-stroke diesel engine employing nanolubricants. Particuology 37: 54-63 (2018)

[183] Kheireddin B A, Lu W, Chen I-C, Akbulut M. Inorganic nanoparticle-based ionic liquid lubricants. Wear 303: 185190 (2013)

[184] Peña-Parás L, Taha-Tijerina J, García A, Maldonado D, González J A, Molina D, Palacios E, Cantú P. Antiwear and extreme pressure properties of nanofluids for industrial applications. Tribol Trans 57: 1072-1076 (2014)

[185] Rahmati B, Sarhan A A, Sayuti M. Morphology of surface generated by end milling AL6061-T6 using molybdenum disulfide $\left(\mathrm{MoS}_{2}\right)$ nanolubrication in end milling machining. J Clean Prod 66: 685-691 (2014)

[186] Duan G, Hu X, Song X, Qiu Z, Gong H, Cao B. Morphology evolution of $\mathrm{ZnO}$ submicroparticles induced by laser irradiation and their enhanced tribology properties by compositing with $\mathrm{Al}_{2} \mathrm{O}_{3}$ nanoparticles. Adv Eng Mater 17: 341-348 (2015)

[187] Konicek A R, Jacobs P W, Webster M N, Schilowitz A M. Role of tribofilms in wear protection. Tribol Int 94: 14-19 (2016)

[188] Ali M K A, Xianjun H, Essa F, Abdelkareem M A A, Elagouz A, Sharshir S W. Friction and wear reduction mechanisms of the reciprocating contact interfaces using nanolubricant under different loads and speeds. $J$ Tribol 140: 051606 (2018)

[189] Ali M K A, Xianjun H, Turkson R F, Peng Z, Chen X. Enhancing the thermophysical properties and tribological behaviour of engine oils using nano-lubricant additives. RSC Adv 6: 77913-77924 (2016)

[190] Ali M K A, Hou X, Mai L, Chen B, Turkson R F, Cai Q. Reducing frictional power losses and improving the scuffing resistance in automotive engines using hybrid nanomaterials as nano-lubricant additives. Wear 364-365: 270-281 (2016)

[191] Turkson R F, Yan F, Ali M K A, Liu B, Hu J. Modeling and Multi-objective optimization of engine performance and hydrocarbon emissions via the use of a computer aided engineering code and the NSGAII genetic algorithm. Sustainability 8: 72 (2016)

[192] Gustavsson F, Jacobson S. Diverse mechanisms of friction induced self-organisation into a low-friction material - An overview of $\mathrm{WS}_{2}$ tribofilm formation. Tribol Int 101: 340-347 (2016)

[193] Kim S Ch, Mansurov Yu N, Li S H. The method for producing copper nanoparticles and analysis of their lubricating ability. Solid State Phenomena 265: 738-744 (2017)

[194] Du P, Chen G, Song S, Zhu D, Wu J, Chen P, Chen H. Preparation and tribological properties of $\mathrm{Cu}$-doped muscovite composite particles as lubricant additive. Chem Res Chin 
Univ 33: 430-435 (2017)

[195] Hu C, Bai M, Lv J, Li X. Molecular dynamics simulation of mechanism of nanoparticle in improving load-carrying capacity of lubricant film. Comput Mater Sci 109: 97-103 (2015)

[196] Hu C, Bai M, Lv J, Kou Z, Li X. Molecular dynamics simulation on the tribology properties of two hard nanoparticles (diamond and silicon dioxide) confined by two iron blocks. Tribol Int 90: 297-305 (2015)

[197] Hu C, Bai M, Lv J, Liu H, Li X. Molecular dynamics investigation of the effect of copper nanoparticle on the solid contact between friction surfaces. Appl Surf Sci 321: 302-309 (2014)

[198] Alimirzaloo V, Qaleh S S G, Keshtiban P M, Ahmadi S.

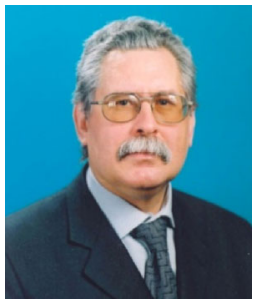

Igor E. UFLYAND. He is the Head of Chemistry Department, Southern Federal University, Rostov-on-Don, Russia. He was born in 1956 and graduated from Rostov State University, Department of Physical and Colloid Chemistry. Prof. Uflyand

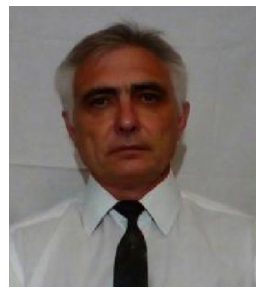

Vladimir A. ZHINZHILO. He graduated from Rostov State Pedagogical University and worked there as an assistant in the Department of Chemistry. Currently, he is an assistant professor in the Depart-

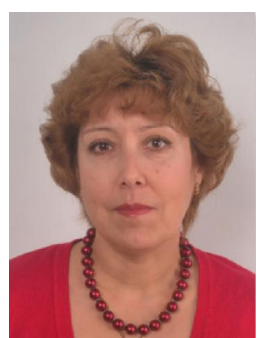

Victoria E. BURLAKOVA. She is the head of Chemistry Department, Don State Technical University, Rostov-on-Don, Russia. She graduated from North Ossetian State University, Faculty of Chemistry, in
Investigation of the effect of $\mathrm{CuO}$ and $\mathrm{Al}_{2} \mathrm{O}_{3}$ nanolubricants on the surface roughness in the forging process of aluminum alloy. Proc Inst Mech Eng Part J: J Eng Tribol 231: 15951604 (2017)

[199] He X, Xiao H, Kyle J P, Terrell E J, Liang H. Twodimensional nanostructured $\mathrm{Y}_{2} \mathrm{O}_{3}$ particles for viscosity modification. Appl Phys Lett 104: 163107 (2014)

[200] He X, Xiao H, Choi H, Díaz A, Mosby B, Clearfield A, Liang H. $\alpha$-Zirconium phosphate nanoplatelets as lubricant additives Colloids Surf A: Physicochem Eng Asp 452: 32 (2014)

[201] Xiao H, Dai W, Kan Y, Clearfield A, Liang H. Amineintercalated $\alpha$-zirconium phosphates as lubricant additives. Appl Surf Sci 329: 384-389 (2015)

received his Ph.D. degree in 1981 and his Doctorate in Chemistry in 1996 from Rostov State University. He became Professor in 1996. His interests include coordination and organometallic chemistry, metal chelate monomers and polymers on their basis, catalysis by metal complexes, tribochemistry, and nanomaterials.

ment of Chemistry at Southern Federal University. His research interests are related to the synthesis of monomeric and polymeric metal chelates, the preparation of nanostructured materials, and their use as additives to lubricants.

1982. Prof. Burlakova received her Ph.D. degree in 1993 from Rostov State University and her Doctorate in Engineering from Don State Technical University in 2006. She became Professor in 2006. Her research interests include triboelectrochemistry, tribotechnical nanomaterials, and electrochemistry. 